\title{
ANALYSIS OF THE ROSENBLATT PROCESS
}

\author{
Ciprian A. TUdor ${ }^{1}$
}

\begin{abstract}
We analyze the Rosenblatt process which is a selfsimilar process with stationary increments and which appears as limit in the so-called Non Central Limit Theorem (Dobrushin and Majòr (1979), Taqqu (1979)). This process is non-Gaussian and it lives in the second Wiener chaos. We give its representation as a Wiener-Itô multiple integral with respect to the Brownian motion on a finite interval and we develop a stochastic calculus with respect to it by using both pathwise type calculus and Malliavin calculus.
\end{abstract}

Mathematics Subject Classification. 60G12, 60G15, 60H05, 60H07.

Received March 3, 2007. Accepted June 4, 2007.

\section{INTRODUCTION}

A selfsimilar object is exactly or approximately similar to a part of itself. Selfsimilar processes are invariant in distribution under suitable scaling. They are of considerable interest in practice since aspects of the selfsimilarity appear in different phenomena like telecommunications, economics, hydrology or turbulence. We refer to the work of Taqqu [44] for a guide on the appearance of the selfsimilarity in many applications and to the monographs by Samorodnitsky and Taqqu [40] and by Embrechts and Maejima [13] for complete expositions on selfsimilar processes.

In this work we analyze a special class of selfsimilar processes that are limits in the so called Non Central Limit Theorem (see Dobrushin and Majòr [11] or Taqqu [43]). Let us briefly recall the general context.

Consider $\left(\xi_{n}\right)_{n \in \mathbb{Z}}$ a stationary Gaussian sequence with mean zero and variance 1 such that its correlation function satisfies

$$
r(n):=\mathbf{E}\left(\xi_{0} \xi_{n}\right)=n^{\frac{2 H-2}{k}} L(n)
$$

with $H \in\left(\frac{1}{2}, 1\right)$ and $L$ is a slowly varying function at infinity (see e.g. [13]). Denote by $H_{m}(x)$ the Hermite polynomial of degree $m$ given by $H_{m}(x)=(-1)^{m} e^{\frac{x^{2}}{2}} \frac{d^{m}}{\mathrm{~d} x^{m}} e^{-\frac{x^{2}}{2}}$. Let $g$ be a function such that $\mathbf{E}\left(g\left(\xi_{0}\right)\right)=0$ and $\mathbf{E}\left(g\left(\xi_{0}\right)^{2}\right)<\infty$. Suppose that $g$ has Hermite rank equal to $k$; that is, if $g$ admits the following expansion in Hermite polynomials

$$
g(x)=\sum_{j \geq 0} c_{j} H_{j}(x), \quad c_{j}=\frac{1}{j !} \mathbf{E}\left(g\left(\xi_{0} H_{j}\left(\xi_{0}\right)\right)\right)
$$

Keywords and phrases. Non Central Limit Theorem, Rosenblatt process, fractional Brownian motion, stochastic calculus via regularization, Malliavin calculus, Skorohod integral.

1 SAmos/MATISSE, Centre d'Économie de La Sorbonne, Université de Panthéon-Sorbonne Paris 1, 90, rue de Tolbiac, 75634 Paris cedex 13, France; Ciprian.Tudor@univ-paris1.fr 
then

$$
k=\min \left\{j ; c_{j} \neq 0\right\} .
$$

Since $\mathbf{E}\left[g\left(\xi_{0}\right)\right]=0$, we have $k \geq 1$. Then the Non Central Limit Theorem $[11,43]$ says that

$$
\frac{1}{n^{H}} \sum_{j=1}^{[n t]} g\left(\xi_{j}\right)
$$

converges as $n \rightarrow \infty$ in the sense of finite dimensional distributions to the process

$$
Z_{H}^{k}(t)=c(H, k) \int_{\mathbb{R}^{k}} \int_{0}^{t}\left(\prod_{j=1}^{k}\left(s-y_{i}\right)_{+}^{-\left(\frac{1}{2}+\frac{1-H}{k}\right)}\right) \mathrm{d} s \mathrm{~d} B\left(y_{1}\right) \ldots \mathrm{d} B\left(y_{k}\right),
$$

where $x_{+}=\max (x, 0)$ and the above integral is a multiple Wiener-Itô stochastic integral with respect to a Brownian motion $B(y))_{y \in \mathbb{R}}$ (see [29] for the definition). The constant $c(H, k)$ is positive and it will be taken such that $\mathbf{E}\left(Z_{H}^{k}(1)^{2}\right)=1$. The process $\left(Z_{H}^{k}(t)\right)_{t \geq 0}$ is called the Hermite process and it is $H$-selfsimilar in the sense that for any $c>0,\left(Z_{H}^{k}(c t)\right)={ }^{(d)}\left(c^{H} Z_{H}^{k}(t)\right)$, where " $={ }^{(d)}$ " means equivalence of all finite dimensional distributions, and it has stationary increments.

When $k=1$ the process given by (2) is nothing else that the fractional Brownian motion (fBm) with Hurst parameter $H \in\left(\frac{1}{2}, 1\right)$. For $k \geq 2$ the process is not Gaussian. If $k=2$ then the process $(2)$ is known as the Rosenblatt process (it was actually been named in this way by Taqqu in [42]).

The fractional Brownian motion is of course the most studied process in the class of Hermite processes due to its significant importance in modeling. A stochastic calculus with respect to it has been intensively developed in the last decade. We refer, among others, to $[4,5,9,16]$.

Our main interest consists here in the study, from the stochastic calculus point of view, of the Rosenblatt process. Although it received a less important attention than the fractional Brownian motion, this process is still of interest in practical applications because of its self-similarity, stationarity of increments and longrange dependence. Actually the very large utilization of the fractional Brownian motion in practice (hydrology, telecommunications) are due to these properties; one prefers in general $\mathrm{fBm}$ before other processes because it is Gaussian and the calculus for it is easier; but in concrete situations when the gaussianity is not plausible for the model, one can use use for example the Rosenblatt process. There exists a consistent literature that focuses on different theoretical aspects of the Rosenblatt processes. Let us recall some of these works. For example, extremal properties of the Rosenblatt distribution have been studied by J.M. Albin in [2] and [3]. The rate of convergence to the Rosenblatt process in the Non Central Limit Theorem has been given by Leonenko and Ahn [23]. Pipiras [31] and Pipiras and Abry [1] studied the wavelet-type expansion of the Rosenblatt process. A law of iterated logarithm has been given in [15].

Among the applications of the Rosenblatt process in statistics or econometrics, we mention the following:

- in the unit root testing problem with errors being nonlinear transforms of linear processes with longrange dependence, the asymptotic distributions in the model are shown in [47] to be functionals of Hermite processes;

- limiting distributions of the parabolically rescaled solutions of the heat equation with singular nonGaussian data have similar behavior to the Rosenblatt distribution (see [24]);

- the Rosenblatt distribution also appears to be the asymptotic distribution of an estimator related to the semiparametric bootstrap approach to hypothesis tests (see [18]) or to the estimation of the long-range dependence parameter [21].

Besides these more or less practical applications of the Rosenblatt process, denoted in the sequel by $Z$, our motivation is also theoretical; it comes from the recent intensive interest to push further the stochastic calculus with respect to more and more general integrator processes. We believe that this process constitutes an interesting and instructive example where the recent developed techniques of the generalized stochastic calculus can 
find a significant test bench. We also mention that, since the process is not Gaussian, several new ideas are needed to develop the calculus for it (for example to prove its stochastic integral form on a finite interval or to use divergence type calculus).

We actually use the two principal methods to develop a stochastic integration theory: the pathwise type calculus and the Malliavin calculus/Skorohod integration. The first approach (that includes essentially the rough paths analysis, see [35], and the stochastic calculus via regularization, see [37]) can be directly applied to the Rosenblatt process because of its regular paths and of the nice covariance structure; a pathwise Itô formula can be written and Stratonovich stochastic equation with $Z$ as noise can be considered. The Malliavin calculus and the Skorohod integration are in general connected in a deeper way to the Gaussian structure of the integrator process and as it will be seen in the present work, Skorohod Itô formula can be derived in a complete form only in particular cases. Although the formula we obtain is rather complicated and not easily tractable, the principal signification of the result is the fact that one can precisely see here that the Gaussian nature of the integrator process is decisive in the stochastic integration theory; once we go out from the Gaussian context, one cannot obtain Itô's formulas that end by a second derivative term.

We would like to mention that some of our results can be directly proved for the more general class of Hermite processes of arbitrary order. For example, the construction of the Wiener integrals or the stochastic calculus via regularization. But in some other places the fact that the process lives in the second Wiener chaos is important and the extension of the results to a higher order Wiener chaos needs a detailed and careful analysis; for example the Skorohod type calculus or the integral representation as a multiple integral with respect to the Brownian motion with a finite time horizon.

We organized our paper as follows. Section 2 presents basic properties of the Rosenblatt process. In particular we prove a stochastic integral representation on a finite integral that will be useful for the construction of the stochastic calculus. In Section 3 we introduce Wiener integrals with respect to $Z$ by following the ideas in [22,25]. We define in Section 4 the Hilbert-valued Rosenblatt process and we consider stochastic evolution equations with this process as noise. Section 5 describes the application of the stochastic calculus via regularization introduced by Russo and Vallois in [37] to the Rosenblatt process and in Section 6 we discuss the Skorohod (divergence) integral: we define the integral and we give conditions that ensure the integrability and the continuity of the indefinite integral process. In Section 7 we prove the relation between the pathwise and the divergence integrals: here the pathwise integral is equal to the Skorohod integral plus two trace terms (in the fBm case there is only a trace term). Finally Section 8 contains a discussion on the Itô formula in the Skorohod sense.

\section{On the Rosenblatt process}

In this section we will analysis some basic properties of the Rosenblatt process; in particular we are interested in its representation as a stochastic integral on a finite interval. As we said, this process is obtained by taking $k=2$ in the relation $(2)$, so

$$
Z_{2}(t):=Z(t)=a(H) \int_{\mathbb{R}} \int_{\mathbb{R}}\left(\int_{0}^{t}\left(s-y_{1}\right)_{+}^{-\frac{2-H}{2}}\left(s-y_{2}\right)_{+}^{-\frac{2-H}{2}} \mathrm{~d} s\right) \mathrm{d} B\left(y_{1}\right) \mathrm{d} B\left(y_{2}\right)
$$

where $(B(y), y \in \mathbb{R})$ is a standard Brownian motion on $\mathbb{R}$. The constant $a(H)$ is a positive normalizing constant and it is chosen such that $\mathbf{E}\left(Z(1)^{2}\right)=1$. It follows actually from [25] that

$$
a(H)^{2}=\left(\frac{\beta\left(\frac{H}{2}, H-1\right)^{2}}{2 H(2 H-1)}\right)^{-1}
$$

Recall that the process $(Z(t))_{t \in[0, T]}$ is selfsimilar of order $H$ and it has stationary increments; it admits a Hölder continuous version of order $\delta<H$. Since $H \in\left(\frac{1}{2}, 1\right)$, it follows that the process $Z$ exhibits long-range dependence. 
Since our main interest consists in the construction of the stochastic calculus with respect to the process $Z$, the representation (3) is not very convenient; as in the fBm case, we would like to represent $Z_{t}$ as a stochastic integral with respect to a Brownian motion with time interval $[0, T]$. Recall that the fBm with $H>\frac{1}{2}$ can be written as

$$
B_{t}^{H}=\int_{0}^{t} K^{H}(t, s) \mathrm{d} W_{s}
$$

with $\left(W_{t}, t \in[0, T]\right)$ a standard Wiener process and

$$
K^{H}(t, s)=c_{H} s^{\frac{1}{2}-H} \int_{s}^{t}(u-s)^{H-\frac{3}{2}} u^{H-\frac{1}{2}} \mathrm{~d} u
$$

where $t>s$ and

$$
c_{H}=\left(\frac{H(2 H-1)}{\beta\left(2-2 H, H-\frac{1}{2}\right)}\right)^{\frac{1}{2}} .
$$

Note that to prove the representation (4) (at least in law) it suffices to see that the right member has the same covariance $R$ as the $\mathrm{fBm}$; otherwise, it can be easily seen from the expression of the kernel $K$ that the right member in (4) is $H$-selfsimilar with stationary increments and as a consequence it cannot be nothing else but a fractional Brownian motion with parameter $H$.

Since the Rosenblatt process is not Gaussian, the proof in its case of a similar representation to (4) needs a supplementary argument; in fact we have the following

Proposition 1. Let $K$ be the kernels (5) and let $(Z(t))_{t \in[0, T]}$ be a Rosenblatt process with parameter $H$. Then it holds that

$$
Z(t)={ }^{(d)} \mathrm{d}(H) \int_{0}^{t} \int_{0}^{t}\left[\int_{y_{1} \vee y_{2}}^{t} \frac{\partial K^{H^{\prime}}}{\partial u}\left(u, y_{1}\right) \frac{\partial K^{H^{\prime}}}{\partial u}\left(u, y_{2}\right) \mathrm{d} u\right] \mathrm{d} B\left(y_{1}\right) \mathrm{d} B\left(y_{2}\right)
$$

where $\left(B_{t}, t \in[0, T]\right)$ is a Brownian motion,

and

$$
H^{\prime}=\frac{H+1}{2}
$$

$$
\mathrm{d}(H)=\frac{1}{H+1}\left(\frac{H}{2(2 H-1)}\right)^{-\frac{1}{2}} \text {. }
$$

\section{Remark 1.}

i) As far as we know the above representation has not been previously proved. This fact is not surprising since even the corresponding representation of the fBm by using the kernel $K^{H}$ is rather new (it is due to [27]). For the sake of completeness, we present a proof of the Proposition 1 in the Appendix.

ii) The constant $\mathrm{d}(H)$ is a normalizing constant, it has been chosen such that $\mathbf{E}(Z(t) Z(s))=$ $\frac{1}{2}\left(t^{2 H}+s^{2 H}-|t-s|^{2 H}\right)$. Indeed,

$$
\begin{aligned}
\mathbf{E}(Z(t) Z(s))= & 2 \mathrm{~d}(H)^{2} \int_{0}^{t \wedge s} \int_{0}^{t \wedge s} \mathrm{~d} y_{1} \mathrm{~d} y_{2} \\
& \times\left(\int_{y_{1} \vee y_{2}}^{t} \int_{y_{1} \vee y_{2}}^{s} \frac{\partial K^{H^{\prime}}}{\partial u}\left(u, y_{1}\right) \frac{\partial K^{H^{\prime}}}{\partial u}\left(u, y_{2}\right) \frac{\partial K^{H^{\prime}}}{\partial u}\left(v, y_{1}\right) \frac{\partial K^{H^{\prime}}}{\partial v}\left(v, y_{2}\right) \mathrm{d} u \mathrm{~d} v\right) \\
= & 2 \mathrm{~d}(H)^{2} \int_{0}^{t} \int_{0}^{s} \mathrm{~d} u \mathrm{~d} v\left(\int_{0}^{u \wedge v} \frac{\partial K^{H^{\prime}}}{\partial u}\left(u, y_{1}\right) \frac{\partial K^{H^{\prime}}}{\partial u}\left(v, y_{1}\right) \mathrm{d} y_{1}\right)^{2} \\
= & 2 \mathrm{~d}(H)^{2}\left(H^{\prime}\left(2 H^{\prime}-1\right)\right)^{2} \int_{0}^{t} \int_{0}^{s}|u-v|^{2 H-2} \mathrm{~d} u \mathrm{~d} v=R(t, s) .
\end{aligned}
$$


iii) It can be seen without without difficulty that the process

$$
Z^{\prime}(t):=\mathrm{d}(H) \int_{0}^{t} \int_{0}^{t}\left[\int_{y_{1} \vee y_{2}}^{t} \frac{\partial K^{H^{\prime}}}{\partial u}\left(u, y_{1}\right) \frac{\partial K^{H^{\prime}}}{\partial u}\left(u, y_{2}\right) \mathrm{d} u\right] \mathrm{d} B\left(y_{1}\right) \mathrm{d} B\left(y_{2}\right)
$$

defines a $H$ selfsimilar process with stationary increments. Indeed, for any $c>0$,

$$
\begin{aligned}
Z^{\prime}(c t) & =\int_{0}^{c t} \int_{0}^{c t}\left[\int_{y_{1} \vee y_{2}}^{c t} \frac{\partial K^{H^{\prime}}}{\partial u}\left(u, y_{1}\right) \frac{\partial K^{H^{\prime}}}{\partial u}\left(u, y_{2}\right) \mathrm{d} u\right] \mathrm{d} B\left(y_{1}\right) \mathrm{d} B\left(y_{2}\right) \\
& =\int_{0}^{c t} \int_{0}^{c t}\left[\int_{\frac{y_{1}}{c} \vee \frac{y_{2}}{c}}^{t} \frac{\partial K^{H^{\prime}}}{\partial u}\left(c u, y_{1}\right) \frac{\partial K^{H^{\prime}}}{\partial u}\left(c u, y_{2}\right) c \mathrm{~d} u\right] \mathrm{d} B\left(y_{1}\right) \mathrm{d} B\left(y_{2}\right) \\
& =\int_{0}^{t} \int_{0}^{t}\left[\int_{y_{1} \vee y_{2}}^{t} \frac{\partial K^{H^{\prime}}}{\partial u}\left(c u, c y_{1}\right) \frac{\partial K^{H^{\prime}}}{\partial u}\left(c u, c y_{2}\right) c \mathrm{~d} u\right] \mathrm{d} B\left(c y_{1}\right) \mathrm{d} B\left(c y_{2}\right)
\end{aligned}
$$

and since $B(c y)={ }^{(d)} c^{\frac{1}{2}} B(y)$ and $\frac{\partial K^{H^{\prime}}}{\partial u}\left(c u, c y_{i}\right)=c^{H^{\prime}-\frac{3}{2}} \frac{\partial K^{H^{\prime}}}{\partial u}\left(u, y_{i}\right)$ we obtain $Z(c t)={ }^{(d)} c^{H} Z(t)$.

The fact that $Z^{\prime}$ has stationary increments follows from the relation

$$
K^{H^{\prime}}(t+h, s)-K^{H^{\prime}}(t, s)=K^{H^{\prime}}(t-s, h)
$$

for any $s, t \in[0, T], s<t$ and $h>0$.

From now on we will use the version of the Rosenblatt process given by the right side of (7).

We will finish this section by proving that the Rosenblatt process possesses a similar property to the fBm, that is, it can be approximated by a sequence of semimartingales (here actually, since $H>\frac{1}{2}$, by a sequence of bounded variation processes). In the $\mathrm{fBm}$ case, the property is inherited by the divergence integral (see [4,6,7]); this fact can be used to construct financial models with the Rosenblatt process as noise (see [6]).

The basic observation is that, if one interchanges formally the stochastic and Lebesque integrals in (7), one gets

$$
Z(t) "=" \int_{0}^{t}\left(\int_{0}^{u} \int_{0}^{u} \frac{\partial K^{H^{\prime}}}{\partial u}\left(u, y_{1}\right) \frac{\partial K^{H^{\prime}}}{\partial u}\left(u, y_{2}\right) \mathrm{d} B\left(y_{1}\right) \mathrm{d} B\left(y_{2}\right)\right) \mathrm{d} u
$$

but the above expression cannot hold because the kernel $\frac{\partial K^{H^{\prime}}}{\partial u}\left(u, y_{1}\right) \frac{\partial K^{H^{\prime}}}{\partial u}\left(u, y_{2}\right)$ does not belong to $L^{2}\left([0, T]^{2}\right)$ since the partial derivative $\frac{\partial K^{H^{\prime}}}{\partial u}\left(u, y_{1}\right)$ behaves on the diagonal as $\left(u-y_{1}\right)^{\frac{H-2}{2}}$.

Let us define, for every $\varepsilon>0$,

$$
\begin{aligned}
Z^{\varepsilon}(t) & =\mathrm{d}(H) \int_{0}^{t} \int_{0}^{t}\left[\int_{y_{1} \vee y_{2}}^{t} \frac{\partial K^{H^{\prime}}}{\partial u}\left(u+\varepsilon, y_{1}\right) \frac{\partial K^{H^{\prime}}}{\partial u}\left(u+\varepsilon, y_{2}\right) \mathrm{d} u\right] \mathrm{d} B\left(y_{1}\right) \mathrm{d} B\left(y_{2}\right) \\
& =\int_{0}^{t}\left(\int_{0}^{u} \int_{0}^{u} \frac{\partial K^{H^{\prime}}}{\partial u}\left(u+\varepsilon, y_{1}\right) \frac{\partial K^{H^{\prime}}}{\partial u}\left(u+\varepsilon, y_{2}\right) \mathrm{d} B\left(y_{1}\right) \mathrm{d} B\left(y_{2}\right)\right) \mathrm{d} u \\
& :=\int_{0}^{t} A_{\varepsilon}(u) \mathrm{d} u
\end{aligned}
$$

Since $A_{\varepsilon} \in L^{2}([0, T] \times \Omega)$ for every $\varepsilon>0$ and it is adapted, it follows that the process $Z^{\varepsilon}$ is a semimartingale.

Proposition 2. For every $t \in[0, T], Z^{\varepsilon}(t) \rightarrow Z(t)$ in $L^{2}(\Omega)$. 
Proof. We have

$$
\begin{aligned}
& Z^{\varepsilon}(t)-Z(t)=\int_{0}^{t} \int_{0}^{t} \mathrm{~d} B\left(y_{1}\right) \mathrm{d} B\left(y_{2}\right) \\
& \times\left(\int_{y_{1} \vee y_{2}}^{t}\left(\frac{\partial K^{H^{\prime}}}{\partial u}\left(u+\varepsilon, y_{1}\right) \frac{\partial K^{H^{\prime}}}{\partial u}\left(u+\varepsilon, y_{2}\right)-\frac{\partial K^{H^{\prime}}}{\partial u}\left(u, y_{1}\right) \frac{\partial K^{H^{\prime}}}{\partial u}\left(u, y_{2}\right)\right) \mathrm{d} u\right)
\end{aligned}
$$

and

$$
\begin{aligned}
\mathbf{E}\left|Z^{\varepsilon}(t)-Z(t)\right|^{2}= & 2 \int_{0}^{t} \int_{0}^{t} \mathrm{~d} y_{1} \mathrm{~d} y_{2} \int_{y_{1} \vee y_{2}}^{t} \int_{y_{1} \vee y_{2}}^{t} \mathrm{~d} v \mathrm{~d} u \\
& \times\left(\frac{\partial K^{H^{\prime}}}{\partial u}\left(u+\varepsilon, y_{1}\right) \frac{\partial K^{H^{\prime}}}{\partial u}\left(u+\varepsilon, y_{2}\right)-\frac{\partial K^{H^{\prime}}}{\partial u}\left(u, y_{1}\right) \frac{\partial K^{H^{\prime}}}{\partial u}\left(u, y_{2}\right)\right) \\
& \times\left(\frac{\partial K^{H^{\prime}}}{\partial v}\left(v+\varepsilon, y_{1}\right) \frac{\partial K^{H^{\prime}}}{\partial v}\left(v+\varepsilon, y_{2}\right)-\frac{\partial K^{H^{\prime}}}{\partial v}\left(v, y_{1}\right) \frac{\partial K^{H^{\prime}}}{\partial v}\left(v, y_{2}\right)\right) .
\end{aligned}
$$

Clearly the quantity $\left(\frac{\partial K^{H^{\prime}}}{\partial u}\left(u+\varepsilon, y_{1}\right) \frac{\partial K^{H^{\prime}}}{\partial u}\left(u+\varepsilon, y_{2}\right)-\frac{\partial K^{H^{\prime}}}{\partial u}\left(u, y_{1}\right) \frac{\partial K^{H^{\prime}}}{\partial u}\left(u, y_{2}\right)\right)$ converges to zero as $\varepsilon \rightarrow 0$ for every $u, y_{1}, y_{2}$ and the conclusion follows by the dominated convergence theorem.

\section{WIENER INTEGRALS}

The covariance structure of the Rosenblatt process allows to construct Wiener integrals with respect to it. We refer to Maejima and Tudor [25] for the definition of Wiener integrals with respect to general Hermite processes and to Kruk et al. [22] for a more general context. Let us recall the main points and translate this construction in our context.

One note that

$$
Z(t)=\int_{0}^{T} \int_{0}^{T} I\left(1_{[0, t]}\right)\left(y_{1}, y_{2}\right) \mathrm{d} B\left(y_{1}\right) \mathrm{d} B\left(y_{2}\right)
$$

where the operator $I$ is defined on the set of functions $f:[0, T] \rightarrow \mathbb{R}$ and takes values in the set of functions $g:[0, T]^{2} \rightarrow \mathbb{R}^{2}$ and it is given by

$$
I(f)\left(y_{1}, y_{2}\right)=\mathrm{d}(H) \int_{y_{1} \vee y_{2}}^{T} f(u) \frac{\partial K^{H^{\prime}}}{\partial u}\left(u, y_{1}\right) \frac{\partial K^{H^{\prime}}}{\partial u}\left(u, y_{2}\right) \mathrm{d} u .
$$

If $f$ is an element of the set $\mathcal{E}$ of step functions on $[0, T]$ of the form

$$
f=\sum_{i=0}^{n-1} a_{i} 1_{\left(t_{i}, t_{i+1}\right]}, \quad t_{i} \in[0, T]
$$

then we naturally define its Wiener integral with respect to $Z$ as

$$
\int_{0}^{T} f(u) \mathrm{d} Z(u):=\sum_{i=0}^{n-1} a_{i}\left(Z_{t_{i+1}}-Z_{t_{i}}\right)=\int_{0}^{T} \int_{0}^{T} I(f)\left(y_{1}, y_{2}\right) \mathrm{d} B\left(y_{1}\right) \mathrm{d} B\left(y_{2}\right) .
$$

Let $\mathcal{H}$ be the set of functions $f$ such that

$$
\|f\|_{\mathcal{H}}^{2}:=2 \int_{0}^{T} \int_{0}^{T} I(f)\left(y_{1}, y_{2}\right)^{2} \mathrm{~d} y_{1} \mathrm{~d} y_{2}<\infty
$$


It can be seen that

$$
\begin{aligned}
\|f\|_{\mathcal{H}}^{2}= & 2 \mathrm{~d}(H)^{2} \int_{0}^{T} \int_{0}^{T}\left(\int_{y_{1} \vee y_{2}}^{T} f(u) \frac{\partial K^{H^{\prime}}}{\partial u}\left(u, y_{1}\right) \frac{\partial K^{H^{\prime}}}{\partial u}\left(u, y_{2}\right) \mathrm{d} u\right)^{2} \mathrm{~d} y_{1} \mathrm{~d} y_{2} \\
= & 2 \mathrm{~d}(H)^{2} \int_{0}^{T} \int_{0}^{T} \mathrm{~d} y_{1} \mathrm{~d} y_{2} \int_{y_{1} \vee y_{2}}^{T} \int_{y_{1} \vee y_{2}}^{T} \mathrm{~d} u \mathrm{~d} v \\
& \times f(u) f(v) \frac{\partial K^{H^{\prime}}}{\partial u}\left(u, y_{1}\right) \frac{\partial K^{H^{\prime}}}{\partial u}\left(u, y_{2}\right) \frac{\partial K^{H^{\prime}}}{\partial v}\left(v, y_{1}\right) \frac{\partial K^{H^{\prime}}}{\partial v}\left(v, y_{2}\right) \\
= & 2 \mathrm{~d}(H)^{2} \int_{0}^{T} \int_{0}^{T}\left(\int_{0}^{u \wedge v} \frac{\partial K^{H^{\prime}}}{\partial u}\left(u, y_{1}\right) \frac{\partial K^{H^{\prime}}}{\partial v}\left(v, y_{1}\right) \mathrm{d} y_{1}\right)^{2} \mathrm{~d} u \mathrm{~d} v \\
= & H(2 H-1) \int_{0}^{T} \int_{0}^{T} f(u) f(v)|u-v|^{2 H-2} \mathrm{~d} v \mathrm{~d} u .
\end{aligned}
$$

It can be proved as in [25] or [22] that the mapping

$$
f \rightarrow \int_{0}^{T} f(u) \mathrm{d} Z(u)
$$

defines an isometry from $\mathcal{E}$ to $L^{2}(\Omega)$ and it can be extended by continuity to an isometry from $\mathcal{H}$ to $L^{2}(\Omega)$ because $\mathcal{E}$ is dense in $\mathcal{H}$ (see [33]). We will call this extension the Wiener integral of $f \in \mathcal{H}$ with respect to $Z$.

Remark 2. It follows from Pipiras and Taqqu (see [33]) that the space $\mathcal{H}$ contains not only functions but its elements could be also distributions. Therefore it is suitable to know subspaces of $\mathcal{H}$ that are spaces of functions. A such subspace is $|\mathcal{H}|$ where

$$
|\mathcal{H}|=\left\{f:[0, T] \rightarrow \mathbb{R}\left|\int_{0}^{T} \int_{0}^{T}\right| f(u)|| f(v)|| u-\left.v\right|^{2 H-2} \mathrm{~d} u \mathrm{~d} v<\infty\right\} .
$$

It actually holds

$$
L^{\frac{1}{H}}([0, T]) \subset|\mathcal{H}| \subset \mathcal{H} .
$$

The space $|\mathcal{H}|$ (and hence $\mathcal{H}$ ) is not complete with respect to the norm $\|\cdot\|_{\mathcal{H}}$ but it is a Banach space with respect to the norm

$$
\|f\|_{|\mathcal{H}|}^{2}=H(2 H-1) \int_{0}^{T} \int_{0}^{T}|f(u)\|f(v)\| u-v|^{2 H-2} \mathrm{~d} u \mathrm{~d} v .
$$

The Wiener integrals $\int_{0}^{T} f(u) \mathrm{d} Z(u)$ and $\int_{0}^{T} g(u) \mathrm{d} Z(u)$ are not necessarily independent when the functions $f$ and $g$ are orthogonal in $\mathcal{H}$. A characterization of their independence is given in the next result.

Proposition 3. Let $f, g \in \mathcal{H}$. Then $\int_{0}^{T} f(u) \mathrm{d} Z(u)$ and $\int_{0}^{T} g(u) \mathrm{d} Z(u)$ are independent if and only if

$$
\left\langle f(\cdot) \frac{\partial K^{H^{\prime}}}{\partial u}\left(\cdot, y_{1}\right), g(\cdot) \frac{\partial K^{H^{\prime}}}{\partial u}\left(\cdot, y_{2}\right)\right\rangle_{\mathcal{H}^{\prime}}=0 \quad \text { a.e. }\left(y_{1}, y_{2}\right) \in[0, T]^{2}
$$

where $\mathcal{H}^{\prime}$ is the space analogous to $\mathcal{H}$ corresponding to the Hurst parameter $H^{\prime}$.

Proof. We use a result by Üstunel-Zakai [41] (see also Kallenberg [20]): two multiple Wiener-Itô integrals with respect to the standard Wiener process $I_{n}(f)$ and $I_{m}(g)$ with $f, g$ symmetric, $f \in L^{2}[0, T]^{n}$ and $g \in L^{2}[0, T]^{m}$ 
are independent if and only if $f \otimes_{1} g=0$ a.e. on $[0, T]^{m+n-2}$, where

$$
\left(f \otimes_{1} g\right)\left(t_{1}, \ldots, t_{n-1}, s_{1}, \ldots, s_{m-1}\right)=\int_{0}^{T} f\left(t_{1}, \ldots, t_{n-1}, t\right) g\left(s_{1}, \ldots, s_{n-1}, t\right) \mathrm{d} t .
$$

Let us apply the above result to

$$
F\left(y_{1}, y_{2}\right)=1_{[0, t]^{2}}\left(y_{1}, y_{2}\right) \int_{y_{1} \vee y_{2}}^{T} f(u) \frac{\partial K^{H^{\prime}}}{\partial u}\left(u, y_{1}\right) \frac{\partial K^{H^{\prime}}}{\partial u}\left(u, y_{2}\right) \mathrm{d} u
$$

and

$$
G\left(y_{1}, y_{2}\right)=1_{[0, t]^{2}}\left(y_{1}, y_{2}\right) \int_{y_{1} \vee y_{2}}^{T} g(u) \frac{\partial K^{H^{\prime}}}{\partial u}\left(u, y_{1}\right) \frac{\partial K^{H^{\prime}}}{\partial u}\left(u, y_{2}\right) \mathrm{d} u
$$

Then

$$
\begin{aligned}
\left(F \otimes_{1} G\right)\left(y_{1}, y_{2}\right)= & \int_{0}^{T} \mathrm{~d} s \\
& \times \int_{y_{1} \vee s}^{T} f(u) \frac{\partial K^{H^{\prime}}}{\partial u}\left(u, y_{1}\right) \frac{\partial K^{H^{\prime}}}{\partial u}(u, s) \mathrm{d} u \int_{y_{2} \vee s}^{T} g(v) \frac{\partial K^{H^{\prime}}}{\partial v}\left(v, y_{2}\right) \frac{\partial K^{H^{\prime}}}{\partial v}(v, s) \mathrm{d} v \\
= & c(H) \int_{0}^{T} \int_{0}^{T} f(u) g(v) \frac{\partial K^{H^{\prime}}}{\partial u}\left(u, y_{1}\right) \frac{\partial K^{H^{\prime}}}{\partial v}\left(v, y_{2}\right)|u-v|^{2 H^{\prime}-2} \mathrm{~d} v \mathrm{~d} u
\end{aligned}
$$

and the conclusion follows easily.

As an immediate consequence, we obtain

Corollary 1. If $f \otimes g=0$ a.e. on $[0, T]^{2}$ then the random variables $\int_{0}^{T} f(u) \mathrm{d} Z(u)$ and $\int_{0}^{T} g(u) \mathrm{d} Z(u)$ are independent.

Remark 3. The Non Central Limit Theorem given by $[11,43]$ can be extended to Wiener integrals (see [25]). More precisely, under suitable assumptions on the deterministic function $f \in \mathcal{H}$ one obtains that the sequence

$$
\frac{1}{n^{H}} \sum_{j \in \mathbb{Z}} f\left(\frac{j}{n}\right) g\left(\xi_{j}\right)
$$

converges weakly when $n \rightarrow \infty$, to the Wiener integral $\int f(u) \mathrm{d} Z(u)\left(g\right.$ and $\xi_{j}$ were introduced in Sect. 1).

\section{INFINITE DIMENSIONAL PROCESS AND STOCHASTIC EVOLUTION EQUATIONS}

In this part we define a Hilbert-valued Rosenblatt process and we consider stochastic evolution equations driven by it.

Let us consider $U$ a real and separable Hilbert space and $Q$ a nuclear, self-adjoint positive and nuclear operator on $U$. There exists then a sequence $0<\lambda_{n} \searrow 0$ of eigenvalues of $Q$ such that $\sum_{n>1} \lambda_{n}<\infty$. Moreover the corresponding eigenvectors form an orthonormal basis in $U$. We define the infinite dimensional Rosenblatt process on $U$ as

$$
Z(t)=\sum_{\nu \geq 0} \sqrt{\lambda_{n}} e_{n} z_{j}(t)
$$

where $\left(z_{j}\right)_{j \geq 0}$ is a family of real independent Rosenblatt processes. 
Note that the series (15) is convergent in $L^{2}(\Omega)$ for every $t \in[0, T]$ since

$$
\mathbf{E}|Z(t)|^{2}=\sum_{j \geq 1} \lambda_{j} \mathbf{E}\left(z_{j}^{2}\right)=t^{2 H} \sum_{j \geq 1} \lambda_{j}<\infty .
$$

Note also that $Z$ has covariance function $R(t, s)$ in the sense that for every $u, v \in U$, and for every $s, t \in[0, T]$

$$
\mathbf{E}\langle Z(t), u\rangle_{U}\langle Z(s), v\rangle_{U}=R(t, s)\langle Q u, v\rangle_{U}
$$

This can be proved exactly as fBm case (see [46]).

In some situations the assumption that $Q$ is nuclear is not convenient. For example one cannot take $Q$ to be the identity operator, that is $\lambda_{n}=1$ for every $n$. Therefore, if $\sum_{n} \lambda_{n}=\infty$ we will consider a bigger real and separable Hilbert space $U_{1} \supset U$ such that the inclusion $U \subset U_{1}$ is nuclear. Then the quantity

$$
Z(t)=\sum_{j} z_{j}(t) e_{j}
$$

is well-defined stochastic process in $U_{1}$.

In the sequel we will consider the infinite dimensional Rosenblatt process to be defined by (16).

Following the one dimensional case, one can introduce Wiener integrals with respect to the Hilbert-valued process $Z$. Let $V$ be another Hilbert space Let $\left(\Phi_{s}, s \in[0, T]\right)$ a stochastic process with valued in the space of linear operators $\mathcal{L}(U, V)$. We put for every $t \in[0, T]$

$$
\int_{0}^{t} \Phi_{s} \mathrm{~d} Z(s)=\sum_{j \geq 1} \int_{0}^{t} \Phi_{s} e_{j} \mathrm{~d} Z_{j}(s)
$$

where $\int_{0}^{t} \Phi_{s} e_{j} \mathrm{~d} Z_{j}(s)$ is a $V$ valued random variable. Note that the integral exists in $L^{2}(\Omega, V)$ if

$$
\mathbf{E}\left|\int_{0}^{t} \Phi_{s} \mathrm{~d} Z(s)\right|_{V}^{2}=\sum_{j}\left|\left\|\Phi e_{j}\right\|_{\mathcal{H}}\right|_{V}^{2}<\infty
$$

Remark 4. If the integrand $\Phi$ does not depend on time, then we find

$$
\mathbf{E}\left|\int_{0}^{t} \Phi \mathrm{d} Z(s)\right|_{V}^{2}=\sum_{j}\left|\Phi e_{n}\right|_{V}^{2} \mathbf{E}\left|\int_{0}^{t} \mathrm{~d} z_{j}(s)\right|^{2}=t^{2 H} \sum_{j}\left|\Phi e_{n}\right|_{V}^{2}
$$

and it can be seen that the integral $\int_{0}^{t} \Phi \mathrm{d} Z(s)$ exists if and only if $\Phi$ is a Hilbert-Schmidt operator.

Now we introduce stochastic evolution equations driven by the infinite-dimensional Rosenblatt process. Let $A: \operatorname{Dom}(A) \subset V \rightarrow V$ be the infinitesimal generator of the strongly continuous semigroup $\left(e^{t A}\right)_{t \in[0, T]}$. We study the equation

$$
\mathrm{d} X(t)=A X(t) \mathrm{d} t+\Phi \mathrm{d} Z(t)
$$

where $X(0)=x \in V$ and $\Phi \in \mathcal{L}(U ; V)$. We will consider mild solution of (17), that is, (when it exists), it can be written as

$$
X(t)=\mathrm{e}^{t A} x+\int_{0}^{t} \mathrm{e}^{(t-s) A} \Phi \mathrm{d} Z(s) .
$$


We will not assume that $\Phi$ is Hilbert-Schmidt (although the integral $\int \Phi \mathrm{d} Z$ exists if and only if $\Phi$ is HilbertSchmidt); this assumption is unnecessary because, under suitable hypothesis on $A$, the integral $\int_{0}^{t} \mathrm{e}^{(t-s) A} \Phi \mathrm{d} Z(s)$ will exist even when $\Phi$ is not Hilbert-Schmidt operator.

The method used in the $\mathrm{fBm}$ case will allow to prove the next theorem.

Theorem 1. Let $Z$ be given by (16) with $H \in\left(\frac{1}{2}, 1\right)$. Consider $\Phi \in \mathcal{L}(U ; V)$ and $A: \operatorname{Dom}(A) \subset V \rightarrow V$ be a negative self-adjoint operator. Then there exists a mild solution $X$ of the equation (17) if and only if the operator $\Phi^{\star} G_{H}(-A) \Phi$ is a trace class operator, where

$$
G_{H}(\lambda)=(\max (\lambda, 1))^{-2 H} .
$$

Remark 5. In [45] in the fBm case is assumed that the spectrum of $A, \sigma(A) \subset-(\infty,-l]$ with $l>0$. The situation when 0 is an accumulation point of the spectrum is not treated; this case is solved in [12].

Proof. The proof is similar as in [45] because the covariance structure of the Rosenblatt process is identical to the one of the fractional Brownian motion.

If $\mathbb{S}^{1}$ denotes the unit circle and $A$ is the Laplacian on the circle, we have

Corollary 2. Assume that $U=V=L^{2}\left(\mathbb{S}^{1}\right)$ and $A=\Delta$ is the Laplacian on $U$. Denote by $\left(e_{n}, f_{n}\right)_{n \geq 1}$ the eigenvectors of $\Delta$ that form an orthonormal basis in $L^{2}\left(\mathbb{S}^{1}\right)$. Let $\left(q_{n}\right)_{n}$ be a bounded sequence of non-negative real numbers and

$$
Z(t)=\sum_{n} \sqrt{q_{n}} e_{n} z_{n}(t)+\sum_{n} \sqrt{q_{n}} f_{n} \tilde{z}_{n}(t)
$$

with $\left(z_{j}, \tilde{z}_{j}\right)_{j}$ independent real Rosenblatt processes. Then (17) has an unique mild solution such that $X(t) \in$ $L^{2}(\Omega, V)$ if an only if

$$
\sum_{n} q_{n} n^{-4 H}<\infty
$$

\section{Pathwise stochastic Calculus}

At this point, we will start to develop a stochastic integration theory with respect to the Rosenblatt process. In general, for processes that are not semimartingales, Itô's theory cannot be applied. One needs generalized alternative ways to integrate stochastically with respect to such processes. In general these generalized method are essentially of two types: the first is the pathwise type calculus and (here we included the rough path analysis [35] and the stochastic calculus via regularization [37]) and the second type is Malliavin calculus and the Skorohod integration theory [29]. In general the pathwise type calculus is connected to the trajectorial regularity and/or the covariance structure of the integrator process. The Malliavin calculus instead is very related to the Gaussian character of the driven process.

Since the Rosenblatt process with $H>\frac{1}{2}$ has zero quadratic variation (see [38]) and regular paths (Hölder continuous of order $H-\varepsilon$ ), the pathwise calculus can be naturally applied to construct stochastic integrals with respect to it. Here we choose to use the approach of Russo and Vallois. Let us list first the main ingredients of the stochastic calculus via regularization.

Let $\left(X_{t}\right)_{t \geq 0}$ and $\left(Y_{t}\right)_{t \geq 0}$ continuous processes. We introduce, for every $t$,

$$
\begin{gathered}
I^{-}(\varepsilon, Y, \mathrm{~d} X)=\int_{0}^{t} Y_{s} \frac{X_{s+\varepsilon}-X_{s}}{\varepsilon} \mathrm{d} s, I^{+}(\varepsilon, Y, \mathrm{~d} X)=\int_{0}^{t} Y_{s} \frac{X_{s}-X_{(s-\varepsilon)_{+}}}{\varepsilon} \mathrm{d} s, \\
I^{0}(\varepsilon, Y, \mathrm{~d} X)=\int_{0}^{t} Y_{s} \frac{X_{s+\varepsilon}-X_{(s-\varepsilon)_{+}}}{2 \varepsilon} \mathrm{d} s
\end{gathered}
$$

and

$$
C_{\varepsilon}(X, Y)(t)=\int_{0}^{t} \frac{\left(X_{s+\varepsilon}-X_{(s-\varepsilon)_{+}}\right)\left(Y_{s+\varepsilon}-Y_{(s-\varepsilon)_{+}}\right)}{\varepsilon} \mathrm{d} s .
$$


Then the forward, backward and symmetric integrals of $Y$ with respect to $X$ will be given

$$
\int_{0}^{t} Y \mathrm{~d}^{-} X=\lim _{\varepsilon \rightarrow 0^{+}} I^{-}(\varepsilon, Y, \mathrm{~d} X), \quad \int_{0}^{t} Y d^{+} X=\lim _{\varepsilon \rightarrow 0^{+}} I^{+}(\varepsilon, Y, \mathrm{~d} X),
$$

and

$$
\int_{0}^{t} Y \mathrm{~d}^{0} X=\lim _{\varepsilon \rightarrow 0^{+}} I^{0}(\varepsilon, Y, \mathrm{~d} X)
$$

provided that the above limits exist uniformly in probability (ucp). The covariation of $X$ and $Y$ is defined as

$$
[X, Y]_{t}=u c p-\lim _{\varepsilon \rightarrow 0^{+}} C_{\varepsilon}(X, Y)(t) .
$$

If $X=Y$ we denote $[X, X]=[X]$ and when $[X]$ exists then $X$ is said to be a finite quadratic variation process. When $[X]=0$, then $X$ is called a zero quadratic variation process.

The Rosenblatt process is clearly a zero quadratic variation process since

$$
\mathbf{E} C_{\varepsilon}(Z, Z)(t)=\mathbf{E} \int_{0}^{t} \frac{1}{\varepsilon}\left(X_{s+\varepsilon}-X_{s}\right)^{2} \mathrm{~d} s=t \varepsilon^{2 H-1} \rightarrow_{\varepsilon \rightarrow 0} 0 .
$$

Therefore the stochastic calculus via regularization can be directly applied to it. Precisely, it follows from Proposition 4.2 of the Russo and Vallois survey [39] that every $f \in C^{2}(\mathbb{R})$, the integrals

$$
\int_{0}^{t} f^{\prime}(X) \mathrm{d}^{-} X, \quad \int_{0}^{t} f^{\prime}(X) \mathrm{d}^{+} X, \quad \int_{0}^{t} f^{\prime}(X) \mathrm{d}^{0} X
$$

exist and are equal and we have the Itô's formula

$$
f\left(X_{t}\right)=f\left(X_{0}\right)+\int_{0}^{t} f^{\prime}(X) \mathrm{d}^{0} X
$$

Remark 6. An immediate consequence of the existence of the quadratic variation of the Rosenblatt process is the existence and uniqueness of the solution of a Stratonovich stochastic differential equation driven by $Z$ (see $[38]$ and see also [28]). Concretely, if $\sigma: \mathbb{R} \rightarrow \mathbb{R}$ and $b:[0, T] \times \mathbb{R} \rightarrow \mathbb{R}$ satisfy some regularity assumptions and $V$ is a locally bounded variation process, then the equation

$$
\mathrm{d} X(t)=\sigma(X(t)) \mathrm{d}^{0} Z(t)+b(t, X(t)) \mathrm{d} V(t)
$$

with $X(0)=G$ where $G$ is an arbitrary random variable, has an unique solution (see [38] for the definition of the solution).

\section{Skorohod integral With Respect to the Rosenblatt PRocess}

In this part we define a divergence integral with respect to $(Z(t))_{t \in[0, T]}$. Constructing generalized Skorohod integrals with respect to processes that are not necessarily Gaussian or semimartingales constitutes a frequent topic. For results in this direction, we refer among others, to [17,19,26,34], or [22].

We will need some basic elements of the Malliavin calculus with respect to a Wiener process $\left(W_{t}\right)_{t \in[0, T]}$. A complete exposition can be found in e.g. [29]. By $\mathcal{S}$ we denote the class of smooth random variables of the form

$$
F=f\left(W_{t_{1}}, \ldots, W_{t_{n}}\right), \quad t_{1}, \ldots, t_{n} \in[0, T]
$$


where $f \in C_{b}^{\infty}\left(\mathbb{R}^{n}\right)$. If $F$ is of the form (23), its Malliavin derivative is defined as

$$
D_{t} F=\sum_{i=1}^{n} \frac{\partial f}{\partial x_{i}}\left(W_{t_{1}}, \ldots, W_{t_{n}}\right) 1_{\left[0, t_{i}\right]}(t), \quad t \in[0, T] .
$$

The operator $D$ is an unbounded closable operator and it can be extended to the closure of $\mathcal{S}$ (denoted $\mathbb{D}^{k, p}$, $k \geq 1$ integer, $p \geq 2$ ) with respect to the norm

$$
\|F\|_{k, p}^{p}=\mathbf{E}|F|^{p}+\sum_{j=1}^{k} \mathbf{E}\left\|D^{(j)} F\right\|_{L^{2}\left([0, T]^{j}\right)}^{p}, \quad F \in \mathcal{S}, k \geq 1, p \geq 2
$$

where the $j$ th derivative $D^{(j)}$ is defined by iteration.

The Skorohod integral $\delta$ is the adjoint of $D$. Its domain is

$$
\operatorname{Dom}(\delta)=\left\{u \in L^{2}([0, T] \times \Omega) /\left|\mathbf{E} \int_{0}^{T} u_{s} D_{s} F \mathrm{~d} s\right| \leq C\|F\|_{2}\right\}
$$

and $D$ and $\delta$ satisfy the duality relationship

$$
\mathbf{E}(F \delta(u))=\mathbf{E} \int_{0}^{T} D_{s} F u_{s} \mathrm{~d} s, \quad F \in \mathcal{S}, u \in \operatorname{Dom}(\delta)
$$

We define $\mathbb{L}^{k, p}=L^{p}\left([0, T] ; \mathbb{D}^{k, p}\right)$. Note that $\mathbb{L}^{k, p} \subset \operatorname{Dom}(\delta)$. We denote $\delta(u)=\int_{0}^{T} u_{s} \delta W_{s}$. We will need the integration by parts formula

if $F \in \mathbb{D}^{1,2}$ and $u \in \mathbb{L}^{1,2}$.

$$
F \delta(u)=\delta(F u)+\int_{0}^{T} D_{s} F u_{s}
$$

It is also possible to define a double anticipating integral with respect to $W$. Basically, a two parameter stochastic process $\left(u_{s, t}\right)_{s, t \in[0, T]}$ is said to be twice Skorohod integrable $\left(u \in \operatorname{Dom}\left(\delta^{(2)}\right)\right)$ if for every $t$ the process $s \rightarrow u(t, s)$ in Skorohod integrable and the process $t \rightarrow \delta(u(t, \cdot))$ is again Skorohod integrable with respect to the Wiener process. The double anticipating integral of $u$ will be denoted by $\delta^{(2)}(u)$.

We also mention that the Skorohod integral with respect to the $\mathrm{fBm} B^{H}$ with Hurst parameter $H>\frac{1}{2}$ is defined through a transfer operator

$$
\int_{0}^{T} g_{s} \mathrm{~d} B_{s}^{H}=\int_{0}^{T} \int_{s}^{T} g_{r} \frac{\partial K^{H}}{\partial r}(r, s) \mathrm{d} r \mathrm{~d} W_{s}
$$

where the integral in the right side above is a Skorohod integral with respect to $W$. Moreover $g$ is Skorohod integrable with respect to $B^{H}$ if the quantity $\int_{s}^{T} g_{r} \frac{\partial K^{H}}{\partial r}(r, s) \mathrm{d} r$ is Skorohod integrable with respect to $W$.

Definition 1. Let us consider a square integrable stochastic process $\left(g_{s}\right)_{s \in[0, T]}$. Following (12) and (26) we define its Skorohod integral with respect to $Z$ by

$$
\begin{aligned}
\int_{0}^{T} g_{s} \mathrm{~d} Z(s) & :=\int_{0}^{T} \int_{0}^{T} I(g)\left(y_{1}, y_{2}\right) \mathrm{d} B\left(y_{1}\right) \mathrm{d} B\left(y_{2}\right) \\
& =\int_{0}^{T} \int_{0}^{T}\left(\int_{y_{1} \vee y_{2}}^{T} g(u) \frac{\partial K^{H^{\prime}}}{\partial u}\left(u, y_{1}\right) \frac{\partial K^{H^{\prime}}}{\partial u}\left(u, y_{2}\right) \mathrm{d} u\right) \mathrm{d} B\left(y_{1}\right) \mathrm{d} B\left(y_{2}\right) .
\end{aligned}
$$


We will say that a process $g$ is Skorohod integrable with respect to $Z$ if the process $I g \in \operatorname{Dom} \delta^{(2)}$, where $\delta^{(2)}$ is the double Skorohod integral with respect to the Brownian motion $B$.

We refer to [30] for the study of double (and multiple) Skorohod integrals.

Remark 7. Note that the Skorohod integral coincides with the Wiener integral if the integrand $g$ is a deterministic function in $\mathcal{H}$. Another Skorohod integral with respect to $Z$ has been introduced in [22] as the adjoint of some Malliavin derivative with respect to $Z$ but this integral does not coincide with the Wiener integral for deterministic integrands.

Next lemma gives a condition that ensures the Skorohod integrability.

Lemma 1. Let $g \in L^{2}(\Omega ; \mathcal{H})$ be such that $g \in \mathbb{L}^{2,2}$ and

$$
\mathbf{E} \int_{0}^{T} \int_{0}^{T}\left\|D_{x_{1}, x_{2}} g\right\|_{\mathcal{H}}^{2} \mathrm{~d} x_{1} \mathrm{~d} x_{2}<\infty .
$$

Then $g$ is Skorohod integrable with respect to $Z$ and

$$
\mathbf{E}\left|\int_{0}^{T} g_{s} \delta Z(s)\right|^{2} \leq c s t .\left[\mathbf{E}\|g\|_{\mathcal{H}}^{2}+\mathbf{E} \int_{0}^{T} \int_{0}^{T}\left\|D_{x_{1}, x_{2}} g\right\|_{\mathcal{H}}^{2} \mathrm{~d} x_{1} \mathrm{~d} x_{2}\right] .
$$

Proof. We use Meyer's inequality for the double Skorohod integral (see [30], p. 320) and we obtain

$$
\begin{aligned}
\mathbf{E}\left|\int_{0}^{T} g_{s} \delta Z(s)\right|^{2} \leq & c s t .\left[\mathbf{E} \int_{0}^{T} \int_{0}^{T} I(g)\left(y_{1}, y_{2}\right)^{2} \mathrm{~d} y_{1} \mathrm{~d} y_{2}\right. \\
& \left.+\mathbf{E} \int_{0}^{T} \int_{0}^{T} \int_{0}^{T} \int_{0}^{T}\left(D_{x_{1}, x_{2}} I(g)\left(y_{1}, y_{2}\right)\right)^{2} \mathrm{~d} x_{1} \mathrm{~d} x_{2} \mathrm{~d} y_{1} \mathrm{~d} y_{2}\right] \\
= & c s t .\left[\mathbf{E} H(2 H-1) \int_{0}^{T} \int_{0}^{T} g(u) g(v)|u-v|^{2 H-2} \mathrm{~d} u \mathrm{~d} v\right. \\
& \left.+\int_{0}^{T} \int_{0}^{T} \mathrm{~d} x_{1} \mathrm{~d} x_{2}\left(\int_{0}^{T} \int_{0}^{T} D_{x_{1}, x_{2}} g(u) D_{x_{1}, x_{2}} g(v)|u-v|^{2 H-2} \mathrm{~d} v \mathrm{~d} u\right)\right] \\
= & \text { cst. }\left[\mathbf{E}\|g\|_{\mathcal{H}}^{2}+\mathbf{E} \int_{0}^{T} \int_{0}^{T}\left\|D_{x_{1}, x_{2}} g\right\|_{\mathcal{H}}^{2} \mathrm{~d} x_{1} \mathrm{~d} x_{2}\right] .
\end{aligned}
$$

Corollary 3. If $g \in L^{2}(\Omega ;|\mathcal{H}|)$ be such that $g \in \mathbb{L}^{2,2}$ and

$$
\mathbf{E} \int_{0}^{T} \int_{0}^{T}\left\|D_{x_{1}, x_{2}} g\right\|_{|\mathcal{H}|}^{2} \mathrm{~d} x_{1} \mathrm{~d} x_{2}<\infty .
$$

Then $g$ is Skorohod integrable with respect to $Z$ and

$$
\mathbf{E}\left|\int_{0}^{T} g_{s} \delta Z(s)\right|^{2} \leq c s t .\|g\|^{2}
$$

where

$$
\|g\|^{2}=\left[\mathbf{E}\|g\|_{|\mathcal{H}|}^{2}+\mathbf{E} \int_{0}^{T} \int_{0}^{T}\left\|D_{x_{1}, x_{2}} g\right\|_{|\mathcal{H}|}^{2} \mathrm{~d} x_{1} \mathrm{~d} x_{2}\right] .
$$


Example 1. The Rosenblatt process $Z$ is Skorohod integrable with respect to $Z$ and

$$
\mathbf{E}\left|\int_{0}^{T} g_{s} \delta Z(s)\right|^{2} \leq c s t . \int_{0}^{T} \int_{0}^{T} R(u, v)|u-v|^{2 H-2} \mathrm{~d} u \mathrm{~d} v .
$$

Proof. We treat the two terms in the right side of (29). Clearly

$$
\mathbf{E}\|Z\|_{\mathcal{H}}^{2}=\text { cst. } \int_{0}^{T} \int_{0}^{T} R(u, v)|u-v|^{2 H-2} \mathrm{~d} u \mathrm{~d} v
$$

We have, for every $x_{1}, x_{2} \in[0, T]$,

$$
D_{x_{1}, x_{2}} Z(u)=2 \mathrm{~d}(H) 1_{[0, u]^{2}}\left(x_{1}, x_{2}\right) \int_{x_{1} \vee x_{2}}^{u} \frac{\partial K^{H^{\prime}}}{\partial u^{\prime}}\left(u^{\prime}, x_{1}\right) \frac{\partial K^{H^{\prime}}}{\partial u^{\prime}}\left(u^{\prime}, x_{2}\right) \mathrm{d} u^{\prime}
$$

and then (note also that $\frac{\partial K^{H^{\prime}}}{\partial t}(t, s)$ is positive and therefore we omit the absolute value at a certain point)

$$
\begin{aligned}
\mathbf{E} \int_{0}^{T} \int_{0}^{T}\left\|D_{x_{1}, x_{2}} g\right\|_{|\mathcal{H}|}^{2} \mathrm{~d} x_{1} \mathrm{~d} x_{2}= & \int_{0}^{T} \int_{0}^{T} \mathrm{~d} x_{1} \mathrm{~d} x_{2} \int_{x_{1} \vee x_{2}}^{T} \int_{x_{1} \vee x_{2}}^{T}|u-v|^{2 H-2} \mathrm{~d} u \mathrm{~d} v \\
& \times \int_{x_{1} \vee x_{2}}^{u} \frac{\partial K^{H^{\prime}}}{\partial u^{\prime}}\left(u^{\prime}, x_{1}\right) \frac{\partial K^{H^{\prime}}}{\partial u^{\prime}}\left(u^{\prime}, x_{2}\right) \mathrm{d} u^{\prime} \int_{x_{1} \vee x_{2}}^{v} \frac{\partial K^{H^{\prime}}}{\partial v^{\prime}}\left(v^{\prime}, x_{1}\right) \frac{\partial K^{H^{\prime}}}{\partial v^{\prime}}\left(v^{\prime}, x_{2}\right) \mathrm{d} v^{\prime} \mid \\
& =\int_{0}^{T} \int_{0}^{T}|u-v|^{2 H-2} \mathrm{~d} u \mathrm{~d} v \int_{0}^{u} \int_{0}^{v}\left(\int_{0}^{u^{\prime} \wedge v^{\prime}} \frac{\partial K^{H^{\prime}}}{\partial u^{\prime}}\left(u^{\prime}, x_{1}\right) \frac{\partial K}{\partial v^{\prime}}\left(v^{\prime}, x_{1}\right) \mathrm{d} x_{1}\right)^{2} \\
& =c s t . \int_{0}^{T} \int_{0}^{T} R(u, v)|u-v|^{2 H-2} \mathrm{~d} u \mathrm{~d} v .
\end{aligned}
$$

We finish the section by a result on the continuity of the indefinite Skorohod integral process. This shows that the indefinite integral keeps the same order of Hölder regularity as the Rosenblatt process.

Proposition 4. Let $g \in \mathbb{L}^{2, p}$ such that

$$
\sup _{r}\left\|g_{r}\right\|_{2, p} \leq \infty
$$

Then the indefinite Skorohod integral process $\left(X_{t}=\int_{0}^{t} g_{s} \delta Z(s), t \in[0, T]\right)$ admits a Hölder continuous version of order $\delta<H$.

Proof. We can write

$$
\begin{aligned}
X_{t}-X_{s}= & \int_{s}^{t} \int_{s}^{t}\left(\int_{y_{1} \vee y_{2}}^{t} \frac{\partial K^{H^{\prime}}}{\partial u}\left(u, y_{1}\right) \frac{\partial K^{H^{\prime}}}{\partial u}\left(u, y_{2}\right) \mathrm{d} u\right) \mathrm{d} B\left(y_{1}\right) \mathrm{d} B\left(y_{2}\right) \\
& +2 \int_{0}^{s} \int_{s}^{t}\left(\int_{y_{2}}^{t} \frac{\partial K^{H^{\prime}}}{\partial u}\left(u, y_{1}\right) \frac{\partial K^{H^{\prime}}}{\partial u}\left(u, y_{2}\right) \mathrm{d} u\right) \mathrm{d} B\left(y_{1}\right) \mathrm{d} B\left(y_{2}\right) \\
& +\int_{0}^{s} \int_{0}^{s}\left(\int_{s}^{t} \frac{\partial K^{H^{\prime}}}{\partial u}\left(u, y_{1}\right) \frac{\partial K^{H^{\prime}}}{\partial u}\left(u, y_{2}\right) \mathrm{d} u\right) \mathrm{d} B\left(y_{1}\right) \mathrm{d} B\left(y_{2}\right) \\
:= & J_{1}+2 J_{2}+J_{3} .
\end{aligned}
$$


Then

By Meyer's inequality [30], p. 320)

$$
\mathbf{E}\left|X_{t}-X_{s}\right|^{p} \leq c(p) \mathbf{E}\left(J_{1}^{p}+J_{2}^{p}+J_{3}^{p}\right)
$$

$$
\begin{aligned}
\mathbf{E}\left|J_{1}\right|^{p} \leq & c(p)\left|\int_{s}^{t} \int_{s}^{t} \mathbf{E}\left(\int_{y_{1} \vee y_{2}}^{t} g_{u} \frac{\partial K^{H^{\prime}}}{\partial u}\left(u, y_{1}\right) \frac{\partial K^{H^{\prime}}}{\partial u}\left(u, y_{2}\right) \mathrm{d} u\right)^{2} \mathrm{~d} y_{1} \mathrm{~d} y_{2}\right|^{\frac{p}{2}} \\
& +c(p) \mathbf{E}\left|\int_{s}^{t} \int_{s}^{t} \int_{0}^{t} \int_{0}^{t}\left[D_{x_{1}, x_{2}} \int_{y_{1} \vee y_{2}}^{t} g_{u} \frac{\partial K^{H^{\prime}}}{\partial u}\left(u, y_{1}\right) \frac{\partial K^{H^{\prime}}}{\partial u}\left(u, y_{2}\right) \mathrm{d} u\right]^{2} \mathrm{~d} x_{1} \mathrm{~d} x_{2} \mathrm{~d} y_{1} \mathrm{~d} y_{2}\right|^{\frac{p}{2}} \\
= & c(p, H)\left|\mathbf{E} \int_{s}^{t} \int_{s}^{t}\right| g(u) g(v)|| u-\left.\left.v\right|^{2 H-2}\right|^{\frac{p}{2}} \\
& +c(p, H) \mathbf{E}\left|\int_{0}^{t} \int_{0}^{t} \mathrm{~d} x_{1} \mathrm{~d} x_{2} \int_{s}^{t} \int_{s}^{t}\right| D_{x_{1}, x_{2}} g_{u} D_{x_{1}, x_{2}} g_{v} \| u-\left.\left.v\right|^{2 H-2} \mathrm{~d} u \mathrm{~d} v\right|^{\frac{p}{2}} \\
\leq & c(p, H) \sup _{r}\left\|g_{r}\right\|_{2, p}^{p}\left|\int_{s}^{t} \int_{s}^{t}\right| u-\left.\left.v\right|^{2 H-2} \mathrm{~d} v \mathrm{~d} u\right|^{\frac{p}{2}} \\
= & c(p, H) \sup _{r}\left\|g_{r}\right\|_{2, p}^{p}(t-s)^{p H} .
\end{aligned}
$$

In a similar way, we can find the same bound for the terms $J_{2}$ and $J_{3}$ (see also [4], proof of Prop. 1). The conclusion will following by the Kolmogorov's continuity criterium.

\section{The Relation Between the Pathwise And the Skorohod integrals}

Let $g$ a stochastic process. Recall that its forward integral with respect to $Z$ is the limit ucp as $\varepsilon \rightarrow 0$ of

$$
I^{-}(\varepsilon, g, \mathrm{~d} Z)=\frac{1}{\varepsilon} \int_{0}^{T} g_{s}(Z(s+\varepsilon)-Z(s)) \mathrm{d} s=\frac{1}{\varepsilon} \int_{0}^{T} g_{s} \delta^{(2)}\left(f_{s+\varepsilon}(\cdot, *)-f_{s}(\cdot, *)\right) \mathrm{d} s
$$

where the kernel $f_{s}$ is given by

$$
f_{s}(x, y)=\mathrm{d}(H) 1_{[0, s]^{2}}(x, y) \int_{x \vee y}^{s} \frac{\partial K^{H^{\prime}}}{\partial u}(u, x) \frac{\partial K^{H^{\prime}}}{\partial u}(u, y) \mathrm{d} u .
$$

We will need the following integration by parts formula (see [30]): if $F \in \mathbb{D}^{2,2}, u \in L^{2}\left([0, T]^{2} \times \Omega\right)$ such that for every $s, u(\cdot, s) \in \operatorname{Dom}(\delta)$, then $F u \in \operatorname{Dom}\left(\delta^{(2)}\right)$ and

$$
F \delta^{(2)}(u)=\delta^{(2)}(F u)+2 \int_{0}^{T} D_{\alpha} F \delta(u(\cdot, \alpha)) \mathrm{d} \alpha-\int_{0}^{T} \int_{0}^{T} D_{\alpha, \beta}^{(2)} F u(\alpha, \beta) \mathrm{d} \alpha \mathrm{d} \beta .
$$

We apply relation $(34)$ to $(32)$ and we obtain

$$
\begin{aligned}
I^{-}(\varepsilon, g, \mathrm{~d} Z)= & \frac{1}{\varepsilon} \int_{0}^{T} \delta^{(2)}\left(g_{s}\left(f_{s+\varepsilon}(\cdot, *)-f_{s}(\cdot, *)\right)\right) \mathrm{d} s \\
& +\frac{2}{\varepsilon} \int_{0}^{T} \int_{0}^{T} D_{\alpha} g_{s} \delta\left(f_{s+\varepsilon}(\cdot, \alpha)-f_{s}(\cdot, \alpha)\right) \mathrm{d} \alpha \mathrm{d} s \\
& -\frac{1}{\varepsilon} \int_{0}^{T} \int_{0}^{T} \int_{0}^{T} D_{\alpha, \beta}^{(2)} g_{s}\left(f_{s+\varepsilon}(\beta, \alpha)-f_{s}(\beta, \alpha)\right) \mathrm{d} \beta \mathrm{d} \alpha \mathrm{d} s .
\end{aligned}
$$


We can already observe, besides the first divergence type term, the appearance of two trace terms. Recall that in the $\mathrm{fBm}$ case the corresponding term $I^{-}\left(\varepsilon, g, \mathrm{~d} B^{H}\right)$ can be decomposed in a divergence term plus a only a trace term.

Definition 2. We say that a stochastic process $g \in \mathbb{L}^{1,2}$ admits a trace of order 1 if

$$
\frac{1}{\varepsilon} \int_{0}^{T} \int_{0}^{T} D_{\alpha} g_{s} \delta\left(f_{s+\varepsilon}(\cdot, \alpha)-f_{s}(\cdot, \alpha)\right) \mathrm{d} \alpha \mathrm{d} s
$$

converges in probability as $\varepsilon \rightarrow 0$. The limit will be denoted by $\operatorname{Tr}^{(1)}\left(D^{(1)} g\right)$.

We say that a stochastic process $g \in \mathbb{L}^{2,2}$ admits a trace of order 2 if

$$
\frac{1}{\varepsilon} \int_{0}^{T} \int_{0}^{T} \int_{0}^{T} D_{\alpha, \beta}^{(2)} g_{s}\left(f_{s+\varepsilon}(\beta, \alpha)-f_{s}(\beta, \alpha)\right) \mathrm{d} \beta \mathrm{d} \alpha \mathrm{d} s
$$

converges in probability as $\varepsilon \rightarrow 0$. The limit will be denoted by $\operatorname{Tr}^{(2)}\left(D^{(2)} g\right)$.

We have the following relation between the divergence and the pathwise integral.

Theorem 2. Let $g \in \mathbb{L}^{2,2}$ such that

$$
\mathbf{E}\|g\|_{|\mathcal{H}|}^{2}+\mathbf{E} \int_{0}^{T} \int_{0}^{T}\left\|D_{x_{1}, x_{2}} g\right\|_{|\mathcal{H}|}^{2} \mathrm{~d} x_{1} \mathrm{~d} x_{2}<\infty .
$$

Assume that $g$ has traces of order 1 and 2. Then $g$ is forward integrable with respect to $Z$ and it holds

$$
\int_{0}^{T} g_{s} \mathrm{~d}^{-} Z(s)=\int_{0}^{T} g_{s} \delta Z(s)+2 \operatorname{Tr}^{(1)}\left(D^{(1)} g\right)-\operatorname{Tr}^{(2)}\left(D^{(2)} g\right) .
$$

Proof. See the Appendix.

\section{On the Itô Formula in the SKOrohod SEnse}

We study Itô's formula for the Rosenblatt process in the divergence sense. As we mentioned before, the Gaussian nature of the integrator process is essential in the framework of the divergence calculus and this fact can be entirely observed here. We state an Itô formula for the Rosenblatt process where we could observe the presence of "the expected terms" (first and second derivatives terms) and a new term involving higher order derivatives. We are actually able to compute this new term in the Itô formula only in particular cases; but more relevant than these formulas, which are not easily tractable, is the fact that one can observe from the computations contained here that the standard method to obtain divergence type change of variables formulas (see e.g. [29]) does not work here, in the sense that one cannot hope to obtain Itô's formulas that stop at $f^{\prime \prime}$.

We will deduce the Skorohod Itô formula by using the pathwise Itô formula. Recall that for any function $f \in C^{2}(\mathbb{R})$

$$
\begin{aligned}
f(Z(t)) & =f(0)+\int_{0}^{t} f^{\prime}(Z(s)) \mathrm{d}^{-} Z(s) \\
& =f(0)+\int_{0}^{t} f^{\prime}(Z(s)) \delta Z(s)+2 T r^{(1)}\left(D^{(1)} f^{\prime}(Z(s))\right)-\operatorname{Tr}^{(2)}\left(D^{(2)} f^{\prime \prime}(Z(s))\right)
\end{aligned}
$$

provided that the above terms exist.

We will now analyze the two trace terms appearing in the above formula. 


\subsection{The trace of order 1}

Recall that

$$
\operatorname{Tr}^{(1)}\left(D^{(1)} f^{\prime}(Z(s))\right)=u c p-\lim _{\varepsilon \rightarrow 0} B_{\varepsilon}
$$

where

$$
\begin{aligned}
B_{\varepsilon} & =\frac{1}{\varepsilon} \int_{0}^{t} \mathrm{~d} s \int_{0}^{t} \mathrm{~d} \alpha D_{\alpha} f^{\prime}(Z(s)) \delta\left(f_{s+\varepsilon}(\cdot, \alpha)-s_{s}(\cdot, \alpha)\right) \\
& =\frac{1}{\varepsilon} \int_{0}^{t} \mathrm{~d} s \int_{0}^{t} \mathrm{~d} \alpha f^{\prime \prime}(Z(s)) D_{\alpha} Z(s) \delta\left(f_{s+\varepsilon}(\cdot, \alpha)-f_{s}(\cdot, \alpha)\right) .
\end{aligned}
$$

The Malliavin derivative of $Z(s)$ is given by

$$
D_{\alpha} Z(s)=2 \mathrm{~d}(H) 1_{[0, s]}(\alpha)\left(\int_{0}^{s}\left(\int_{\alpha \vee y_{1}}^{s} \frac{\partial K^{H^{\prime}}}{\partial u}(u, \alpha) \frac{\partial K^{H^{\prime}}}{\partial u}\left(u, y_{1}\right) \mathrm{d} u\right) \mathrm{d} B\left(y_{1}\right)\right)
$$

Thus

$$
\begin{aligned}
B_{\varepsilon}= & \frac{2}{\varepsilon} \mathrm{d}(H) \int_{0}^{T} \mathrm{~d} s f^{\prime \prime}(Z(s)) \int_{0}^{s} \delta\left(f_{s+\varepsilon}(\cdot, \alpha)-f_{s}(\cdot, \alpha)\right) \mathrm{d} \alpha \\
& \times \int_{0}^{s}\left(\int_{\alpha \vee y_{1}}^{s} \frac{\partial K^{H^{\prime}}}{\partial u}(u, \alpha) \frac{\partial K^{H^{\prime}}}{\partial u}\left(u, y_{1}\right) \mathrm{d} u\right) \mathrm{d} B\left(y_{1}\right)
\end{aligned}
$$

where $f_{s}$ is given by (33). By using the integration by parts formula (25) it holds that

$$
\begin{aligned}
B_{\varepsilon}= & \frac{2}{\varepsilon} \mathrm{d}(H) \int_{0}^{T} \mathrm{~d} s f^{\prime \prime}(Z(s)) \int_{0}^{s} \mathrm{~d} \alpha \\
& \times \int_{0}^{s}\left[\delta\left(f_{s+\varepsilon}(\cdot, \alpha)-f_{s}(\cdot, \alpha)\right) \int_{\alpha \vee y_{1}}^{s} \frac{\partial K^{H^{\prime}}}{\partial u}(u, \alpha) \frac{\partial K^{H^{\prime}}}{\partial u}\left(u, y_{1}\right) \mathrm{d} u\right] \mathrm{d} B\left(y_{1}\right) \\
& +\frac{2}{\varepsilon} \mathrm{d}(H) \int_{0}^{T} \mathrm{~d} s f^{\prime \prime}(Z(s)) \int_{0}^{s} \mathrm{~d} \alpha \\
& \times \int_{0}^{s}\left[\int_{\alpha \vee y_{1}}^{s} \frac{\partial K^{H^{\prime}}}{\partial u}(u, \alpha) \frac{\partial K^{H^{\prime}}}{\partial u}\left(u, y_{1}\right) \mathrm{d} u\left(f_{s+\varepsilon}\left(y_{1}, \alpha\right)-f_{s}\left(y_{1}, \alpha\right)\right] \mathrm{d} y_{1}\right. \\
:= & B_{\varepsilon}^{1}+B_{\varepsilon}^{2} .
\end{aligned}
$$


We regard first the term $B_{\varepsilon}^{2}$ because it can be treated in the same manner for any function $f$. We can write

$$
\begin{aligned}
B_{\varepsilon}^{2}= & \frac{2}{\varepsilon} \mathrm{d}(H)^{2} \int_{0}^{T} \mathrm{~d} s f^{\prime \prime}(Z(s)) \int_{0}^{s} \mathrm{~d} \alpha \\
& \times \int_{0}^{s} \mathrm{~d} y_{1} \int_{\alpha \vee y_{1}}^{s} \frac{\partial K^{H^{\prime}}}{\partial u}(u, \alpha) \frac{\partial K^{H^{\prime}}}{\partial u}\left(u, y_{1}\right) \mathrm{d} u \\
& \times\left[1_{[0, s+\varepsilon]^{2}}\left(y_{1}, \alpha\right) \int_{\alpha \vee y_{1}}^{s+\varepsilon} \frac{\partial K^{H^{\prime}}}{\partial v}(v, \alpha) \frac{\partial K^{H^{\prime}}}{\partial v}\left(v, y_{1}\right) \mathrm{d} v\right. \\
& \left.-1_{[0, s]^{2}}\left(y_{1}, \alpha\right) \int_{\alpha \vee y_{1}}^{s} \frac{\partial K^{H^{\prime}}}{\partial v}(v, \alpha) \frac{\partial K^{H^{\prime}}}{\partial v}\left(v, y_{1}\right) \mathrm{d} v\right] \\
= & \frac{2}{\varepsilon} \mathrm{d}(H)^{2} \int_{0}^{T} \mathrm{~d} s f^{\prime \prime}(Z(s)) \int_{0}^{s} \mathrm{~d} u \int_{s}^{s+\varepsilon} \mathrm{d} v\left(\int_{0}^{u \wedge v} \frac{\partial K^{H^{\prime}}}{\partial u}(u, \alpha) \frac{\partial K^{H^{\prime}}}{\partial v}(v, \alpha) \mathrm{d} \alpha\right)^{2} \\
= & 2 A(H)^{2} \int_{0}^{t} \mathrm{~d} v \int_{0}^{v} \mathrm{~d} u|u-v|^{2 H-2} \frac{1}{\varepsilon} \int_{(v-\varepsilon) \vee u}^{v} f^{\prime \prime}(Z(s)) \mathrm{d} s
\end{aligned}
$$

with $A(H)=H^{\prime}\left(2 H^{\prime}-1\right) \mathrm{d}(H)$ and we have

$$
\begin{aligned}
B_{\varepsilon}^{2}= & 2 A(H)^{2} \int_{0}^{t} \mathrm{~d} v \int_{0}^{v} \mathrm{~d} u|u-v|^{2 H-2}\left(\frac{1}{\varepsilon} \int_{(v-\varepsilon)}^{v} f^{\prime \prime}(Z(s)) \mathrm{d} s\right) \\
& +2 A(H)^{2} \int_{0}^{t} \mathrm{~d} v \int_{v-\varepsilon}^{v} \mathrm{~d} u|u-v|^{2 H-2}\left(\frac{1}{\varepsilon} \int_{u}^{v} f^{\prime \prime}(Z(s)) \mathrm{d} s\right) .
\end{aligned}
$$

Therefore we have the convergence in $L^{1}(\Omega)$ as $\varepsilon \rightarrow 0$

$$
B_{\varepsilon}^{2} \rightarrow 2 A(H)^{2} \int_{0}^{T} \int_{0}^{u} f^{\prime \prime}(Z(v))|u-v|^{2 H-2} \mathrm{~d} v \mathrm{~d} u=H \int_{0}^{T} \int_{0}^{T} f^{\prime \prime}(Z(u)) u^{2 H-1} \mathrm{~d} u
$$

since the first summand in (42) converges to the limit and the second one goes to zero by the dominated convergence theorem.

The study of the term $B^{1}$ is rather difficult to be done in general. We will study it in some particular cases.

\subsection{The trace of order 2}

Recall that

$$
\operatorname{Tr}^{(2)}\left(D^{(2)} f^{\prime}(Z(s))\right)=u c p-\lim _{\varepsilon \rightarrow 0} C_{\varepsilon}
$$

where

$$
\begin{aligned}
C_{\varepsilon}= & \frac{1}{\varepsilon} \int_{0}^{t} \mathrm{~d} s \int_{0}^{t} \int_{0}^{t} D_{\alpha, \beta} f^{\prime}(Z(s))\left(f_{s+\varepsilon}(\alpha, \beta)-f_{s}(\alpha, \beta)\right) \mathrm{d} \alpha \mathrm{d} \beta \\
= & \frac{1}{\varepsilon} \int_{0}^{t} \mathrm{~d} s \int_{0}^{t} \int_{0}^{t}\left(f_{s+\varepsilon}(\alpha, \beta)-f_{s}(\alpha, \beta)\right) \mathrm{d} \alpha \mathrm{d} \beta \\
& \times\left[f^{\prime \prime}(Z(s)) D_{\alpha, \beta} Z(s)+f^{\prime \prime \prime}(Z(s)) D_{\alpha} Z(s) D_{\beta} Z(s)\right] \\
:= & C_{\varepsilon}^{1}+C_{\varepsilon}^{2} .
\end{aligned}
$$


We can write

$$
\begin{aligned}
C_{\varepsilon}^{1} & =\frac{2}{\varepsilon} \mathrm{d}(H)^{2} \int_{0}^{t} f^{\prime \prime}(Z(s)) \mathrm{d} s \int_{0}^{s} \mathrm{~d} u \int_{s}^{s+\varepsilon} \mathrm{d} v\left(\int_{0}^{u \wedge v} \frac{\partial K^{H^{\prime}}}{\partial u}(u, \alpha) \frac{\partial K^{H^{\prime}}}{\partial v}(v, \alpha) \mathrm{d} \alpha\right)^{2} \\
& =\frac{2}{\varepsilon} \mathrm{d}(H)^{2}\left(H^{\prime}\left(2 H^{\prime}-1\right)\right)^{2} \int_{0}^{t} f^{\prime \prime}(Z(s)) \mathrm{d} s \int_{0}^{s} \mathrm{~d} u \int_{s}^{s+\varepsilon} \mathrm{d} v|u-v|^{2 H-2} \\
& =2 \mathrm{~d}(H)^{2}\left(H^{\prime}\left(2 H^{\prime}-1\right)\right)^{2} \int_{0}^{t} \mathrm{~d} v \int_{0}^{v} \mathrm{~d} u|u-v|^{2 H-2}\left(\frac{1}{\varepsilon} \int_{(v-\varepsilon) \vee u}^{v} f^{\prime \prime}(Z(s)) \mathrm{d} s\right)
\end{aligned}
$$

and then clearly

$$
C_{\varepsilon}^{1} \rightarrow H \int_{0}^{t} f^{\prime \prime}(Z(v)) v^{2 H-1} \mathrm{~d} v
$$

in $L^{1}(\Omega)$ as $\varepsilon \rightarrow 0$.

The term denoted by $C_{\varepsilon}^{2}$ can be handled in the following way:

$$
\begin{aligned}
C_{\varepsilon}^{2}= & \frac{1}{\varepsilon} \int_{0}^{t} \mathrm{~d} s f^{\prime \prime \prime}(Z(s)) \int_{0}^{t} \int_{0}^{t} D_{\alpha} Z(s) D_{\beta} Z(s)\left(f_{s+\varepsilon}(\alpha, \beta)-f_{s}(\alpha, \beta)\right) \mathrm{d} \alpha \mathrm{d} \beta \\
= & \frac{4}{\varepsilon} \mathrm{d}(H)^{2} \int_{0}^{t} \mathrm{~d} s f^{\prime \prime \prime}(Z(s)) \int_{0}^{s} \int_{0}^{s} \mathrm{~d} \alpha \mathrm{d} \beta\left(f_{s+\varepsilon}(\alpha, \beta)-f_{s}(\alpha, \beta)\right) \\
& \times\left(\int_{0}^{t} \mathrm{~d} B\left(y_{1}\right) \int_{\alpha \vee y_{1}}^{s} \frac{\partial K^{H^{\prime}}}{\partial u}(u, \alpha) \frac{\partial K^{H^{\prime}}}{\partial u}\left(u, y_{1}\right) \mathrm{d} u\right) \\
& \times\left(\int_{0}^{t} \mathrm{~d} B\left(y_{2}\right) \int_{\beta \vee y_{2}}^{s} \frac{\partial K^{H^{\prime}}}{\partial v}(v, \beta) \frac{\partial K^{H^{\prime}}}{\partial v}\left(v, y_{2}\right) \mathrm{d} v\right) .
\end{aligned}
$$

At this point we can state the following version of the Itô's formula by putting in evidence the first and second derivatives terms plus a new term involving higher order derivatives of the function $f$.

Theorem 3. Let $f \in C^{2}(\mathbb{R})$. Then, for every $t \in[0, T]$

$$
f(Z(t))=f(Z(0))+\int_{0}^{t} f^{\prime}(Z(s)) \delta Z(s)+H \int_{0}^{t} f^{\prime \prime}(Z(s)) s^{2 H-1} \mathrm{~d} s+N_{t}
$$

where $N_{t}=u c p-\lim _{\varepsilon \rightarrow 0}\left(2 B_{\varepsilon}^{1}-C_{\varepsilon}^{2}\right)$ where $B_{\varepsilon}^{1}$ and $C_{\varepsilon}^{2}$ are given by (40) and (45) respectively (provided that the limit exists).

Proof. Is is a consequence of the computations contained in this section.

We will give the explicit expression of the process $N_{t}$ is particular situations.

\subsection{Particular cases}

In this paragraph we compute explicitly the remaining trace term for two particular choices of the function $f$. 
The case $f(x)=x^{2}$.

In this case the term $C_{\varepsilon}^{2}$ clearly vanishes. We will determinate the limit of the term $B_{\varepsilon}^{1}$. We have

$$
\begin{aligned}
B_{\varepsilon}^{1}= & \frac{4}{\varepsilon} \mathrm{d}(H) \int_{0}^{t} \mathrm{~d} s \int_{0}^{s} \mathrm{~d} \alpha \int_{0}^{t} \int_{0}^{t} \mathrm{~d} B\left(y_{1}\right) \mathrm{d} B\left(y_{2}\right) \\
& \times \int_{\alpha \vee y_{1}}^{s} \frac{\partial K^{H^{\prime}}}{\partial u}(u, \alpha) \frac{\partial K^{H^{\prime}}}{\partial u}\left(u, y_{1}\right) \mathrm{d} u\left(f_{s+\varepsilon}\left(y_{2}, \alpha\right)-f_{s}\left(y_{2}, \alpha\right)\right)
\end{aligned}
$$

and by Fubini we get

$$
\begin{aligned}
B_{\varepsilon}^{1}= & 4 \mathrm{~d}(H)^{2} \int_{0}^{t} \int_{0}^{t} \mathrm{~d} B\left(y_{1}\right) \mathrm{d} B\left(y_{2}\right) \int_{y_{2}}^{t} \mathrm{~d} v \int_{y_{1}}^{v} \mathrm{~d} u \frac{\partial K^{H^{\prime}}}{\partial u}\left(u, y_{1}\right) \frac{\partial K^{H^{\prime}}}{\partial u}\left(u, y_{2}\right) \\
& \times\left(\frac{1}{\varepsilon} \int_{(v-\varepsilon) \vee u}^{v} \mathrm{~d} s\right)\left(\int_{0}^{u \wedge v} \frac{\partial K^{H^{\prime}}}{\partial u}(u, \alpha) \frac{\partial K^{H^{\prime}}}{\partial v}(v, \alpha) \mathrm{d} \alpha\right) \\
= & 4 \mathrm{~d}(H)^{2} H^{\prime}\left(2 H^{\prime}-1\right) \int_{0}^{t} \int_{0}^{t} \mathrm{~d} B\left(y_{1}\right) \mathrm{d} B\left(y_{2}\right) \\
& \times \int_{y_{2}}^{t} \mathrm{~d} v \int_{y_{1}}^{v} \mathrm{~d} u \frac{\partial K^{H^{\prime}}}{\partial u}\left(u, y_{1}\right) \frac{\partial K^{H^{\prime}}}{\partial u}\left(u, y_{2}\right)|u-v|^{2 H^{\prime}-2}\left(\frac{1}{\varepsilon} \int_{(v-\varepsilon) \vee u}^{v} \mathrm{~d} s\right)
\end{aligned}
$$

and then one can prove that

$$
B_{\varepsilon}^{1} \rightarrow 4 \mathrm{~d}(H)^{2} H^{\prime}\left(2 H^{\prime}-1\right) \int_{0}^{t} \int_{0}^{t} \mathrm{~d} B\left(y_{1}\right) \mathrm{d} B\left(y_{2}\right) \int_{y_{2}}^{t} \mathrm{~d} v \int_{y_{1}}^{v} \mathrm{~d} u \frac{\partial K^{H^{\prime}}}{\partial u}\left(u, y_{1}\right) \frac{\partial K^{H^{\prime}}}{\partial u}\left(u, y_{2}\right)|u-v|^{2 H^{\prime}-2}
$$

The case $f(x)=x^{3}$. In this case we need to compute both terms $C_{\varepsilon}^{2}$ and $B_{\varepsilon}^{1}$ because they have both non-trivial contributions. Let regard the term (40). It holds by calculating first the integral $\mathrm{d} \alpha$

$$
\begin{aligned}
B_{\varepsilon}^{1}= & \frac{12}{\varepsilon} \mathrm{d}(H)^{2} H^{\prime}\left(2 H^{\prime}-1\right) \int_{0}^{t} Z(s) \mathrm{d} s \\
& \times\left[\int_{0}^{s} \int_{0}^{s+\varepsilon} \mathrm{d} B\left(y_{1}\right) \mathrm{d} B\left(y_{2}\right) \int_{y_{1}}^{s} \mathrm{~d} u \int_{y_{2}}^{s+\varepsilon} \mathrm{d} v \frac{\partial K^{H^{\prime}}}{\partial u}\left(u, y_{1}\right) \frac{\partial K^{H^{\prime}}}{\partial v}\left(v, y_{2}\right)|u-v|^{2 H^{\prime}-2}\right. \\
& -\int_{0}^{s} \int_{0}^{s} \mathrm{~d} B\left(y_{1}\right) \mathrm{d} B\left(y_{2}\right) \int_{y_{1}}^{s} \mathrm{~d} u \int_{y_{2}}^{s} \mathrm{~d} v \frac{\partial K^{H^{\prime}}}{\partial u}\left(u, y_{1}\right) \frac{\partial K^{H^{\prime}}}{\partial v}\left(v, y_{2}\right)|u-v|^{\left.2 H^{\prime}-2\right]}
\end{aligned}
$$

and the integration by parts formula for the double Skorohod integral gives

$$
B_{\varepsilon}^{1}=B_{\varepsilon}^{1,1}+B_{\varepsilon}^{1,2}+B_{\varepsilon}^{1,3}
$$

where

$$
\begin{aligned}
B_{\varepsilon}^{1,1}= & \frac{12}{\varepsilon} \mathrm{d}(H)^{2} H^{\prime}\left(2 H^{\prime}-1\right) \int_{0}^{t} \mathrm{~d} s \\
& \times\left[\int_{0}^{s} \int_{0}^{s+\varepsilon} \mathrm{d} B\left(y_{1}\right) \mathrm{d} B\left(y_{2}\right) Z(s) \int_{y_{1}}^{s} \mathrm{~d} u \int_{y_{2}}^{s+\varepsilon} \mathrm{d} v \frac{\partial K^{H^{\prime}}}{\partial u}\left(u, y_{1}\right) \frac{\partial K^{H^{\prime}}}{\partial v}\left(v, y_{2}\right)|u-v|^{2 H^{\prime}-2}\right. \\
& -\int_{0}^{s} \int_{0}^{s} \mathrm{~d} B\left(y_{1}\right) \mathrm{d} B\left(y_{2}\right) Z(s) \int_{y_{1}}^{s} \mathrm{~d} u \int_{y_{2}}^{s} \mathrm{~d} v \frac{\partial K^{H^{\prime}}}{\partial u}\left(u, y_{1}\right) \frac{\partial K^{H^{\prime}}}{\partial v}\left(v, y_{2}\right)|u-v|^{\left.2 H^{\prime}-2\right]}
\end{aligned}
$$




$$
\begin{aligned}
B_{\varepsilon}^{1,2}= & -\frac{48}{\varepsilon} \mathrm{d}(H)^{3} H^{\prime}\left(2 H^{\prime}-1\right) \int_{0}^{t} \mathrm{~d} s \int_{0}^{s} \mathrm{~d} \alpha \\
& \times \int_{0}^{s}\left(\int_{\alpha \vee y_{1}}^{s} \mathrm{~d} u^{\prime} \frac{\partial K^{H^{\prime}}}{\partial u^{\prime}}\left(u^{\prime}, \alpha\right) \frac{\partial K^{H^{\prime}}}{\partial u^{\prime}}\left(u^{\prime}, y_{1}\right)\right) \mathrm{d} B\left(y_{1}\right) \\
& \times\left[\int_{0}^{s+\varepsilon} \mathrm{d} B\left(y_{2}\right) \int_{\alpha}^{s} \mathrm{~d} u \int_{y_{2}}^{s+\varepsilon} \mathrm{d} v \frac{\partial K^{H^{\prime}}}{\partial u}(u, \alpha) \frac{\partial K^{H^{\prime}}}{\partial v}\left(v, y_{2}\right)|u-v|^{2 H^{\prime}-2}\right. \\
& \left.-\int_{0}^{s} \mathrm{~d} B\left(y_{2}\right) \int_{\alpha}^{s} \mathrm{~d} u \int_{y_{2}}^{s} \mathrm{~d} v \frac{\partial K^{H^{\prime}}}{\partial u}(u, \alpha) \frac{\partial K^{H^{\prime}}}{\partial v}\left(v, y_{2}\right)|u-v|^{2 H^{\prime}-2}\right]
\end{aligned}
$$

and

$$
\begin{aligned}
B_{\varepsilon}^{1,3}= & \frac{24}{\varepsilon} \mathrm{d}(H)^{3} H^{\prime}\left(2 H^{\prime}-1\right) \int_{0}^{t} \mathrm{~d} s \\
& \times\left[\int_{0}^{s} \int_{0}^{s+\varepsilon} \mathrm{d} y_{1} \mathrm{~d} y_{2} \int_{y_{1} \vee y_{2}}^{s} \frac{\partial K^{H^{\prime}}}{\partial u^{\prime}}\left(u^{\prime}, y_{1}\right) \frac{\partial K^{H^{\prime}}}{\partial u^{\prime}}\left(u^{\prime}, y_{2}\right) \mathrm{d} u^{\prime}\right. \\
& \times \int_{y_{1}}^{s} \mathrm{~d} u \int_{y_{2}}^{s+\varepsilon} \mathrm{d} v \frac{\partial K^{H^{\prime}}}{\partial u}\left(u, y_{1}\right) \frac{\partial K^{H^{\prime}}}{\partial v}\left(v, y_{2}\right)|u-v|^{2 H^{\prime}-2} \\
& -\int_{0}^{s} \int_{0}^{s} \mathrm{~d} y_{1} \mathrm{~d} y_{2} \int_{y_{1} \vee y_{2}}^{s} \frac{\partial K^{H^{\prime}}}{\partial u^{\prime}}\left(u^{\prime}, y_{1}\right) \frac{\partial K^{H^{\prime}}}{\partial u^{\prime}}\left(u^{\prime}, y_{2}\right) \mathrm{d} u^{\prime} \\
& \left.\times \int_{y_{1}}^{s} \mathrm{~d} u \int_{y_{2}}^{s} \mathrm{~d} v \frac{\partial K^{H^{\prime}}}{\partial u}\left(u, y_{1}\right) \frac{\partial K^{H^{\prime}}}{\partial v}\left(v, y_{2}\right)|u-v|^{2 H^{\prime}-2}\right] .
\end{aligned}
$$

Now,

$$
\begin{aligned}
B_{\varepsilon}^{1,1}= & 12 \mathrm{~d}(H)^{2} H^{\prime}\left(2 H^{\prime}-1\right) \int_{0}^{t} \int_{0}^{t} \mathrm{~d} B\left(y_{1}\right) \mathrm{d} B\left(y_{2}\right) \\
& \times \int_{y_{2}}^{t} \mathrm{~d} v \int_{y_{1}}^{v} \mathrm{~d} u \frac{\partial K^{H^{\prime}}}{\partial u}\left(u, y_{1}\right) \frac{\partial K^{H^{\prime}}}{\partial v}\left(v, y_{2}\right)|u-v|^{2 H^{\prime}-2}\left(\frac{1}{\varepsilon} \int_{(v-\varepsilon) \vee u}^{v} Z(s) \mathrm{d} s\right)
\end{aligned}
$$

and we have the convergence as in the proof of Theorem 2

$$
B_{\varepsilon}^{1,1} \rightarrow 12 \mathrm{~d}(H)^{2} H^{\prime}\left(2 H^{\prime}-1\right) \int_{0}^{t} \int_{0}^{t} \mathrm{~d} B\left(y_{1}\right) \mathrm{d} B\left(y_{2}\right) \int_{y_{2}}^{t} \mathrm{~d} v \int_{y_{1}}^{v} \mathrm{~d} u Z_{v} \frac{\partial K^{H^{\prime}}}{\partial u}\left(u, y_{1}\right) \frac{\partial K^{H^{\prime}}}{\partial v}\left(v, y_{2}\right)|u-v|^{2 H^{\prime}-2}
$$

To treat the term $B_{\varepsilon}^{1,2}$ one needs to use again the integration by parts formula (25) and one obtains

$$
\begin{aligned}
B_{\varepsilon}^{1,2} \rightarrow & -48 \mathrm{~d}(H)^{3}\left(H^{\prime}\left(2 H^{\prime}-1\right)\right)^{2} \int_{0}^{t} \int_{0}^{t} \mathrm{~d} B\left(y_{1}\right) \mathrm{d} B\left(y_{2}\right) \\
& \times \int_{y_{2}}^{t} \mathrm{~d} v \int_{y_{1}}^{v} \mathrm{~d} u^{\prime} \int_{y_{2}}^{v} \mathrm{~d} u\left|u-u^{\prime}\right|^{2 H^{\prime}-2}|u-v|^{2 H^{\prime}-2} \frac{\partial K^{H^{\prime}}}{\partial u^{\prime}}\left(u^{\prime}, y_{1}\right) \frac{\partial K^{H^{\prime}}}{\partial v}\left(v, y_{2}\right) \\
& -48 \mathrm{~d}(H)^{3}\left(H^{\prime}\left(2 H^{\prime}-1\right)\right)^{3} \int_{0}^{t} \mathrm{~d} v \int_{0}^{v} \int_{0}^{v} \mathrm{~d} u^{\prime} \mathrm{d} u|u-v|^{2 H^{\prime}-2}\left|u^{\prime}-v\right|^{2 H^{\prime}-2}\left|u-u^{\prime}\right|^{2 H^{\prime}-2} .
\end{aligned}
$$


Concerning $B_{\varepsilon}^{1,3}$ we similarly have

$$
B_{\varepsilon}^{1,3} \rightarrow 24 \mathrm{~d}(H)^{3}\left(H^{\prime}\left(2 H^{\prime}-1\right)\right)^{3} \int_{0}^{t} \mathrm{~d} v \int_{0}^{v} \int_{0}^{v} \mathrm{~d} u^{\prime} \mathrm{d} u|u-v|^{2 H^{\prime}-2}\left|u^{\prime}-v\right|^{2 H^{\prime}-2}\left|u-u^{\prime}\right|^{2 H^{\prime}-2} .
$$

Let's study now $C_{\varepsilon}^{2}$. In this case

$$
\begin{aligned}
C_{\varepsilon}^{2}= & \frac{24}{\varepsilon} \mathrm{d}(H)^{2} \int_{0}^{t} \mathrm{~d} s \int_{0}^{s} \int_{0}^{s} \mathrm{~d} \alpha \mathrm{d} \beta\left(f_{s+\varepsilon}(\alpha, \beta)-f_{s}(\alpha, \beta)\right) \\
& \left.\times \int_{0}^{t} \int_{0}^{t} \mathrm{~d} B\left(y_{1}\right) \mathrm{d} B\left(y_{2}\right)\right) \int_{\alpha \vee y_{1}}^{s} \frac{\partial K^{H^{\prime}}}{\partial u}(u, \alpha) \frac{\partial K^{H^{\prime}}}{\partial u}\left(u, y_{1}\right) \mathrm{d} u \int_{\beta \vee y_{2}}^{s} \frac{\partial K^{H^{\prime}}}{\partial v}(v, \beta) \frac{\partial K^{H^{\prime}}}{\partial v}\left(v, y_{2}\right) \mathrm{d} v \\
& +\frac{24}{\varepsilon} \int_{0}^{t} \mathrm{~d} s \int_{0}^{s} \int_{0}^{s} \mathrm{~d} \alpha \mathrm{d} \beta\left(f_{s+\varepsilon}(\alpha, \beta)-f_{s}(\alpha, \beta)\right) \\
& \times \int_{0}^{s} \mathrm{~d} y_{1} \int_{\alpha \vee y_{1}}^{s} \frac{\partial K}{\partial u}(u, \alpha) \frac{\partial K^{H^{\prime}}}{\partial u}\left(u, y_{1}\right) \mathrm{d} u \int_{\beta \vee y_{2}}^{s} \frac{\partial K^{H^{\prime}}}{\partial v}(v, \beta) \frac{\partial K^{H^{\prime}}}{\partial v}\left(v, y_{2}\right) \mathrm{d} v
\end{aligned}
$$

and by the same type of calculations as above we can prove that as $\varepsilon \rightarrow 0$

$$
\begin{aligned}
C_{\varepsilon}^{2} \rightarrow & \left.24 \mathrm{~d}(H)^{3}\left(H^{\prime}\left(2 H^{\prime}-1\right)\right)^{2} \int_{0}^{t} \int_{0}^{t} \mathrm{~d} B\left(y_{1}\right) \mathrm{d} B\left(y_{2}\right)\right) \int_{y_{1}}^{t} \mathrm{~d} u^{\prime} \int_{y_{1}}^{u^{\prime}} \mathrm{d} u \int_{y_{2}}^{u^{\prime}} \mathrm{d} v \\
& \times\left|u-u^{\prime}\right|^{2 H^{\prime}-2}\left|v-u^{\prime}\right|^{2 H^{\prime}-2} \frac{\partial K}{\partial u}\left(u, y_{1}\right) \frac{\partial K^{H^{\prime}}}{\partial v}\left(v, y_{2}\right) \\
& +24 \mathrm{~d}(H)^{3}\left(H^{\prime}\left(2 H^{\prime}-1\right)\right)^{3} \int_{0}^{t} \mathrm{~d} u^{\prime} \int_{0}^{u^{\prime}} \int_{0}^{u^{\prime}} \mathrm{d} u \mathrm{~d} v \\
& \times\left|u-u^{\prime}\right|^{2 H^{\prime}-2}\left|v-u^{\prime}\right|^{2 H^{\prime}-2}|u-v|^{2 H^{\prime}-2}
\end{aligned}
$$

We can summarize

Theorem 4. We have

$$
\begin{aligned}
Z(t)^{2} & =2 \int_{0}^{t} Z(s) \delta Z(s)+t^{2 H} \\
& +\frac{4(2 H-1)}{H+1} \int_{0}^{t} \int_{0}^{t} \mathrm{~d} B\left(y_{1}\right) \mathrm{d} B\left(y_{2}\right) \int_{y_{2}}^{t} \mathrm{~d} v \int_{y_{1}}^{u} \mathrm{~d} u \frac{\partial K^{H^{\prime}}}{\partial u}\left(u, y_{1}\right) \frac{\partial K^{H^{\prime}}}{\partial u}\left(u, y_{2}\right)|u-v|^{2 H^{\prime}-2}
\end{aligned}
$$


and

$$
\begin{aligned}
Z(t)^{3}= & 3 \int_{0}^{t} Z(s)^{2} \delta Z(s)+24 \frac{\left(\mathrm{d}(H) H^{\prime}\left(2 H^{\prime}-1\right)\right)^{2}}{2 H-1} \int_{0}^{t} Z(s) s^{2 H-1} \mathrm{~d} s \\
& +24 \mathrm{~d}(H)^{2} H^{\prime}\left(2 H^{\prime}-1\right) \int_{0}^{t} \int_{0}^{t} \mathrm{~d} B\left(y_{1}\right) \mathrm{d} B\left(y_{2}\right) \\
& \times \int_{y_{2}}^{t} \mathrm{~d} v \int_{y_{1}}^{v} \mathrm{~d} u Z_{v} \frac{\partial K^{H^{\prime}}}{\partial u}\left(u, y_{1}\right) \frac{\partial K^{H^{\prime}}}{\partial v}\left(v, y_{2}\right)|u-v|^{2 H^{\prime}-2} \\
& +72 \mathrm{~d}(H)^{3}\left(H^{\prime}\left(2 H^{\prime}-1\right)\right)^{2} \int_{0}^{t} \int_{0}^{t} \mathrm{~d} B\left(y_{1}\right) \mathrm{d} B\left(y_{2}\right) \\
& \times \int_{y_{2}}^{t} \mathrm{~d} v \int_{y_{1}}^{v} \mathrm{~d} u^{\prime} \int_{y_{1}}^{v} \mathrm{~d} u\left|u-u^{\prime}\right|^{2 H^{\prime}-2}|u-v|^{2 H^{\prime}-2} \frac{\partial K^{H^{\prime}}}{\partial u^{\prime}}\left(u^{\prime}, y_{1}\right) \frac{\partial K^{H^{\prime}}}{\partial v}\left(v, y_{2}\right) \\
& +24 \mathrm{~d}(H)^{3}\left(H^{\prime}\left(2 H^{\prime}-1\right)\right)^{3} \int_{0}^{t} \mathrm{~d} v \int_{0}^{v} \int_{0}^{v} \mathrm{~d} u^{\prime} \mathrm{d} u|u-v|^{2 H^{\prime}-2}\left|u^{\prime}-v\right|^{2 H^{\prime}-2}\left|u-u^{\prime}\right|^{2 H^{\prime}-2} .
\end{aligned}
$$

Remark 8. One can note the appearance of a term involving $f^{\prime \prime \prime}$ in the expression of the summand $C_{\varepsilon}$. Therefore one cannot hope to have Itô's formulas that end with a second derivative term. We believe that it could be very interesting to find the general expression of the Itô formula for the Rosenblatt process. We also feel that a such formula should be very different from the Gaussian case, involving the derivatives of $f$ of any order and the so-called cumulants (see the Appendix, proof of Prop. 1). Actually, the basic difference comes from the fact that the law of a Gaussian process is determined by the covariance function while for processes in a second chaos, the law is determined by the cumulants of any order. Due to this fact, we expect to have such cumulants in the change of variables formula for non-Gaussian processes (their appearance can be noted in the case $f(x)=x^{3}$ ) and also mixtures of multiple Skorohod integrals and deterministic integrals with cumulants.

\section{Appendix}

\subsection{Proof of the Proposition 1}

Let us denote by $Z^{\prime}(t)$ the right hand side of $(7)$. Consider $b_{1}, \ldots, b_{n} \in \mathbb{R}$ and $t_{1}, \ldots, t_{n} \in[0, T]$. We need to show that the random variables

$$
\sum_{l=1}^{n} b_{l} Z\left(t_{l}\right), \quad \sum_{l=1}^{n} b_{l} Z^{\prime}\left(t_{l}\right)
$$

have the same distribution.

We will use the following criterium by Fox and Taqqu (see [14]): If $f \in L^{2}\left([0, T]^{2}\right)$ is a symmetric function, then the law of the multiple Wiener-Itô integral $I_{2}(f)$ is uniquely determined by its cumulants, where the $m$ th cumulant of $f$ is given by

$$
c_{m}(f)=\frac{(m-1) !}{2} 2^{m} \int_{\mathbb{R}^{m}} f\left(x_{1}, x_{2}\right) f\left(x_{2}, x_{3}\right) \ldots f\left(x_{m-1}, x_{m}\right) f\left(x_{m}, x_{1}\right) \mathrm{d} x_{1} \ldots \mathrm{d} x_{m} .
$$

In other words, if two symmetric functions $f, g \in L^{2}\left([0, T]^{2}\right)$ have the same cumulants, then the multiple Wiener-Itô integrals of order two $I_{2}(f)$ and $I_{2}(g)$ have the same law. 
We will show that, for every $t, s \in[0, T]$, the random variables $Z_{t}+Z_{s}$ and $Z_{t}^{\prime}+Z_{s}^{\prime}$ have the same law; the general case case will follow by a similar calculation. It holds that

$$
Z_{t}^{\prime}+Z_{s}^{\prime}=I_{2}\left(f_{t, s}\right)
$$

where

$$
\begin{aligned}
f_{t, s}\left(y_{1}, y_{2}\right)= & 1_{[0, t]}\left(y_{1}\right) 1_{[0, t]}\left(y_{2}\right) \int_{y_{1} \vee y_{2}}^{t} \frac{\partial K^{H^{\prime}}}{\partial u}\left(u, y_{1}\right) \frac{\partial K^{H^{\prime}}}{\partial u}\left(u, y_{2}\right) \mathrm{d} u \\
& +1_{[0, s]}\left(y_{1}\right) 1_{[0, s]}\left(y_{2}\right) \int_{y_{1} \vee y_{2}}^{s} \frac{\partial K^{H^{\prime}}}{\partial u}\left(u, y_{1}\right) \frac{\partial K^{H^{\prime}}}{\partial u}\left(u, y_{2}\right) \mathrm{d} u v .
\end{aligned}
$$

We have denoting by $a_{m}:=\frac{(m-1) !}{2} 2^{m} \mathrm{~d}(H)^{m}$,

$$
\begin{aligned}
c_{m}\left(f_{s, t}\right)= & a(m) \int_{\mathbb{R}^{m}} f_{t, s}\left(y_{1}, y_{2}\right) \ldots f_{t, s}\left(y_{m}, y_{1}\right) \mathrm{d} y_{1} \ldots \mathrm{d} y_{m} \\
= & a(m) \int_{\mathbb{R}^{m}} \mathrm{~d} y_{1} \ldots \mathrm{d} y_{m} \\
& \times\left(\int_{y_{1} \vee y_{2}}^{t} \frac{\partial K^{H^{\prime}}}{\partial u}\left(u_{1}, y_{1}\right) \frac{\partial K^{H^{\prime}}}{\partial u}\left(u_{1}, y_{2}\right) \mathrm{d} u_{1}+\int_{y_{1} \vee y_{2}}^{s} \frac{\partial K^{H^{\prime}}}{\partial u}\left(u_{1}, y_{1}\right) \frac{\partial K^{H^{\prime}}}{\partial u}\left(u_{1}, y_{2}\right) \mathrm{d} u_{1}\right) \\
& \times\left(\int_{y_{2} \vee y_{3}}^{t} \frac{\partial K^{H^{\prime}}}{\partial u}\left(u_{2}, y_{2}\right) \frac{\partial K^{H^{\prime}}}{\partial u}\left(u_{2}, y_{3}\right) \mathrm{d} u_{2}+\int_{y_{2} \vee y_{3}}^{s} \frac{\partial K^{H^{\prime}}}{\partial u}\left(u_{2}, y_{2}\right) \frac{\partial K^{H^{\prime}}}{\partial u}\left(u_{2}, y_{3}\right) \mathrm{d} u_{2}\right) \\
& \left.\times \ldots \int_{y_{m} \vee y_{1}} \frac{\partial K^{H^{\prime}}}{\partial u}\left(u_{m}, y_{m}\right) \frac{\partial K^{H^{\prime}}}{\partial u}\left(u_{m}, y_{1}\right) \mathrm{d} u_{m}+\int_{y_{m} \vee y_{1}} \frac{\partial K^{H^{\prime}}}{\partial u}\left(u_{m}, y_{1}\right) \frac{\partial K^{H^{\prime}}}{\partial u}\left(u_{m}, y_{m}\right) \mathrm{d} u_{m}\right) \\
& \times\left(\int_{y_{m}}^{t}\right.
\end{aligned}
$$

and by classical Fubini theorem

$$
\begin{aligned}
c_{m}\left(f_{s, t}\right)= & a(m) \sum_{t_{j} \in\{t, s\}} \int_{0}^{t_{1}} \ldots \int_{0}^{t_{m}} \mathrm{~d} u_{1} \ldots \mathrm{d} u_{m} \\
& \times\left(\int_{0}^{u_{1} \wedge u_{m}} \frac{\partial K^{H^{\prime}}}{\partial u_{1}}\left(u_{1}, y_{1}\right) \frac{\partial K^{H^{\prime}}}{\partial u_{m}}\left(u_{m}, y_{1}\right) \mathrm{d} y_{1}\right) \\
& \times\left(\int_{0}^{u_{1} \wedge u_{2}} \frac{\partial K^{H^{\prime}}}{\partial u_{1}}\left(u_{1}, y_{2}\right) \frac{\partial K^{H^{\prime}}}{\partial u_{2}}\left(u_{2}, y_{2}\right) \mathrm{d} y_{2}\right) \\
& \ldots \int_{0}^{u_{m-1} \wedge u_{m}} \frac{\partial K^{H^{\prime}}}{\partial u_{m-1}}\left(u_{m}, y_{m}\right) \frac{\partial K^{H^{\prime}}}{\partial u_{m}}\left(u_{m}, y_{m}\right) \mathrm{d} y_{m} \\
= & a(m) \sum_{t_{j} \in\{t, s\}} \int_{0}^{t_{1}} \ldots \int_{0}^{t_{m}} \mathrm{~d} u_{1} \ldots \mathrm{d} u_{m} \\
& \times\left|u_{1}-u_{2}\right|^{2 H^{\prime}-2}\left|u_{2}-u_{3}\right|^{2 H^{\prime}-2} \ldots\left|u_{m}-u_{1}\right|^{2 H^{\prime}-2}
\end{aligned}
$$

with $a(m)=a(m)\left(H^{\prime}\left(2 H^{\prime}-1\right)\right)^{m}$.

The computation of the cumulant of $Z_{t}+Z_{s}$ is similar. Indeed, we can write, for $s, t \in[0, T]$,

$$
Z(t)+Z(s)=I_{2}\left(g_{s, t}\right)
$$


where

$$
g_{s, t}=a(H)\left(\int_{0}^{t}\left(u-y_{1}\right)_{+}^{\frac{H-2}{2}}\left(u-y_{2}\right)_{+}^{\frac{H-2}{2}} \mathrm{~d} u+\int_{0}^{s}\left(u-y_{1}\right)_{+}^{\frac{H-2}{2}}\left(u-y_{2}\right)_{+}^{\frac{H-2}{2}} \mathrm{~d} u\right)
$$

and the $m$ th cumulant of the kernel $g_{s, t}$ is given by

$$
\begin{aligned}
c_{m}\left(g_{s, t}\right)= & b(m) \int_{\mathbb{R}^{m}} \mathrm{~d} y_{1} \ldots \mathrm{d} y_{m} \\
& \times\left(\int_{0}^{t}\left(u_{1}-y_{1}\right)_{+}^{\frac{H-2}{2}}\left(u_{1}-y_{2}\right)_{+}^{\frac{H-2}{2}} \mathrm{~d} u_{1}+\int_{0}^{s}\left(u_{1}-y_{1}\right)_{+}^{\frac{H-2}{2}}\left(u_{1}-y_{2}\right)_{+}^{\frac{H-2}{2}} \mathrm{~d} u_{1}\right) \\
& \times\left(\int_{0}^{t}\left(u_{2}-y_{2}\right)_{+}^{\frac{H-2}{2}}\left(u_{2}-y_{3}\right)_{+}^{\frac{H-2}{2}} \mathrm{~d} u_{2}+\int_{0}^{s}\left(u_{2}-y_{2}\right)_{+}^{\frac{H-2}{2}}\left(u_{2}-y_{3}\right)_{+}^{\frac{H-2}{2}} \mathrm{~d} u_{2}\right) \\
& \cdots \\
& \times\left(\int_{0}^{t}\left(u_{m}-y_{m}\right)_{+}^{\frac{H-2}{2}}\left(u_{m}-y_{1}\right)_{+}^{\frac{H-2}{2}} \mathrm{~d} u_{1}+\int_{0}^{s}\left(u_{m}-y_{m}\right)_{+}^{\frac{H-2}{2}}\left(u_{m}-y_{1}\right)_{+}^{\frac{H-2}{2}} \mathrm{~d} u_{m}\right) \\
= & b(m) \sum_{t_{j} \in\{t, s\}} \int_{0}^{t_{1}} \cdots \int_{0}^{t_{m}} \mathrm{~d} u_{1} \ldots \mathrm{d} u_{m} \\
= & \int_{\mathbb{R}}\left(u_{1}-y_{1}\right)_{+}^{\frac{H-2}{2}}\left(u_{m}-y_{1}\right)_{+}^{\frac{H-2}{2}} \mathrm{~d} y_{1} \int_{\mathbb{R}}\left(u_{1}-y_{2}\right)_{+}^{\frac{H-2}{2}}\left(u_{2}-y_{2}\right)_{+}^{\frac{H-2}{2}} \mathrm{~d} y_{2} \\
& \cdots \int_{\mathbb{R}}\left(u_{m-1}-y_{m}\right)_{+}^{\frac{H-2}{2}}\left(u_{m}-y_{m}\right)_{+}^{\frac{H-2}{2}} \mathrm{~d} y_{m} .
\end{aligned}
$$

Since for any $a>0$

$$
\int_{\mathbb{R}}(u-y)_{+}^{a-1}(v-y)_{+}^{a-1} \mathrm{~d} y=\beta(a, 2 a-1)|u-v|^{2 a-1}
$$

we get

$$
\begin{aligned}
c_{m}\left(g_{s, t}\right)= & b(m) \beta\left(\frac{H}{2}, H-1\right)^{m} \sum_{t_{j} \in\{t, s\}} \int_{0}^{t_{1}} \ldots \int_{0}^{t_{m}} \mathrm{~d} u_{1} \ldots \mathrm{d} u_{m} \\
& \left|u_{1}-u_{2}\right|^{2 H^{\prime}-2}\left|u_{2}-u_{3}\right|^{2 H^{\prime}-2} \ldots\left|u_{m}-u_{1}\right|^{2 H^{\prime}-2}
\end{aligned}
$$

and it remains to observe that $a^{\prime}(m)=b(m)$ which implies that (53) equals (54).

\subsection{Proof of Theorem 2}

By (35) and Definition 2, it suffices to show that the term

$$
A_{\epsilon}=\frac{1}{\varepsilon} \int_{0}^{T} \delta^{(2)}\left(g_{s}\left(f_{s+\varepsilon}(\cdot, *)-f_{s}(\cdot, *)\right)\right) \mathrm{d} s
$$

converges to

$$
\int_{0}^{T} g_{s} \delta Z(s)
$$

in $L^{2}(\Omega)$ as $\varepsilon \rightarrow 0$. 
We can write, by Fubini,

$$
\begin{aligned}
A_{\varepsilon} & =\frac{1}{\varepsilon} \int_{0}^{T} \mathrm{~d} s \int_{0}^{T} \int_{0}^{T} g_{s}\left(f_{s+\varepsilon}\left(y_{1}, y_{2}\right)-f_{s}\left(y_{1}, y_{2}\right)\right) \mathrm{d} B\left(y_{1}\right) \mathrm{d} B\left(y_{2}\right) \\
& =\int_{0}^{T} \int_{0}^{T} \mathrm{~d} B\left(y_{1}\right) \mathrm{d} B\left(y_{2}\right) \int_{y_{1} \vee y_{2}}^{T} g^{\varepsilon}(u) \frac{\partial K^{H^{\prime}}}{\partial u}\left(u, y_{1}\right) \frac{\partial K^{H^{\prime}}}{\partial u}\left(u, y_{2}\right) \mathrm{d} u \\
& =\int_{0}^{T} \int_{0}^{T} I\left(g^{\varepsilon}\right)\left(y_{1}, y_{2}\right) \mathrm{d} B\left(y_{1}\right) \mathrm{d} B\left(y_{2}\right)=\int_{0}^{T} g_{s}^{\varepsilon} \delta Z(s)
\end{aligned}
$$

where we denoted by

$$
g^{\varepsilon}(u)=\frac{1}{\varepsilon} \int_{u-\varepsilon}^{u} g_{s} \mathrm{~d} s .
$$

By using (31), it is sufficient to check that

$$
g^{\varepsilon} \rightarrow_{\varepsilon \rightarrow 0} g \text { in } L^{2}(\Omega ; \mathcal{H})
$$

and

We will show that

$$
\int_{0}^{T} \int_{0}^{T} \mathbf{E}\left\|D_{x_{1}, x_{2}}\left(g^{\varepsilon}-g\right)\right\|_{\mathcal{H}}^{2} \mathrm{~d} x_{1} \mathrm{~d} x_{2} \rightarrow_{\varepsilon \rightarrow 0} 0
$$

and

$$
\left\|g^{\varepsilon}\right\|_{|\mathcal{H}|} \leq c(H)\|g\|_{|\mathcal{H}|}
$$

$$
\int_{0}^{T} \int_{0}^{T}\left\|D_{x_{1}, x_{2}} g^{\varepsilon}\right\|_{|\mathcal{H}|}^{2} \mathrm{~d} x_{1} \mathrm{~d} x_{2} \leq c(H) \int_{0}^{T} \int_{0}^{T}\left\|D_{x_{1}, x_{2}} g\right\|_{|\mathcal{H}|}^{2} \mathrm{~d} x_{1} \mathrm{~d} x_{2} .
$$

The bound (56) has been proved in [5], proof of Proposition 3, Step 1. Concerning the bound (57), we can write

$$
\begin{aligned}
\int_{0}^{T} \int_{0}^{T} \mathbf{E}\left\|D_{x_{1}, x_{2}} g^{\varepsilon}\right\|_{|\mathcal{H}|}^{2} \mathrm{~d} x_{1} \mathrm{~d} x_{2} \\
\quad=c(H) \int_{0}^{T} \int_{0}^{T} \mathrm{~d} x_{1} \mathrm{~d} x_{2} \int_{0}^{T} \int_{0}^{T}\left|D_{x_{1}, x_{2}} g_{u}^{\varepsilon}\right|\left|D_{x_{1}, x_{2}} g_{v}^{\varepsilon}\right||u-v|^{2 H-2} \mathrm{~d} u \mathrm{~d} v \\
\leq c(H) \frac{1}{\varepsilon^{2}} \int_{0}^{T} \int_{0}^{T} \mathrm{~d} x_{1} \mathrm{~d} x_{2} \int_{0}^{T} \int_{0}^{T} \mathrm{~d} u \mathrm{~d} v|u-v|^{2 H-2} \int_{v-\varepsilon}^{v} \int_{u-\varepsilon}^{u} \mathrm{~d} s \mathrm{~d} s^{\prime}\left|D_{x_{1}, x_{2}} g_{s} D_{x_{1}, x_{2}} g_{s^{\prime}}\right| \\
\leq c(H) \int_{0}^{T} \int_{0}^{T} \mathrm{~d} x_{1} \mathrm{~d} x_{2} \int_{0}^{T} \int_{0}^{T} \mathrm{~d} s \mathrm{~d} s^{\prime}\left|D_{x_{1}, x_{2}} g_{s} D_{x_{1}, x_{2}} g_{s^{\prime}}\right|\left(\frac{1}{\varepsilon^{2}} \int_{s}^{s+\varepsilon} \int_{s^{\prime}}^{s^{\prime}+\varepsilon}|u-v|^{2 H-2} \mathrm{~d} u \mathrm{~d} v\right)
\end{aligned}
$$

It follows from [5], proof of Proposition 3, Step 1, that

$$
\frac{1}{\varepsilon^{2}} \int_{s}^{s+\varepsilon} \int_{s^{\prime}}^{s^{\prime}+\varepsilon}|u-v|^{2 H-2} \mathrm{~d} u \mathrm{~d} v \leq c(H)\left|s-s^{\prime}\right|^{2 H-2}
$$

and thus (57) follows.

Now we can finish the proof proceeding as in [5], proof of Proposition 3, Step 3. Consider a sequence $g^{n}$ of simple processes of the form $g^{n}=\sum_{i=0}^{n-1} F_{i} 1_{\left(t_{i}, t_{i+1}\right]}$ with $F_{i} \in \mathcal{S}$ and $t_{i} \in[0, T]$ such that $\left\|g_{n}-g\right\| \rightarrow 0$ in $L^{2}(\Omega)$ when $n \rightarrow \infty$ (the existence of a such sequence follows easily by the densite of $\mathcal{E}$ in $\mathcal{H}$ ). Then by (31) we have that

$$
\int_{0}^{T} g_{s}^{n} \delta Z(s) \rightarrow_{n \rightarrow \infty} \int_{0}^{T} g_{s} \delta Z(s)
$$


Denote by $g^{n, \varepsilon}$ the approximation process of the form (55) associated to $g^{n}$. We can write, for any $\varepsilon>0$ and $n \geq 1$,

$$
\begin{aligned}
& \mathbf{E}\left|\int_{0}^{T} g_{s}^{\varepsilon} \delta Z(s)-\int_{0}^{T} g_{s} \delta Z(s)\right|^{2} \leq 3\left(\mathbf{E}\left|\int_{0}^{T} g_{s}^{\varepsilon} \delta Z(s)-\int_{0}^{T} g_{s}^{n, \varepsilon} \delta Z(s)\right|^{2}\right. \\
& \left.+\mathbf{E}\left|\int_{0}^{T} g_{s}^{n, \varepsilon} \delta Z(s)-\int_{0}^{T} g_{s}^{n} \delta Z(s)\right|^{2}+\mathbf{E}\left|\int_{0}^{T} g_{s}^{n} \delta Z(s)-\int_{0}^{T} g_{s} \delta Z(s)\right|^{2}\right) .
\end{aligned}
$$

By (56) and (57) it follows that for $n$ large enough and for any $\delta>0$

$$
\mathbf{E}\left|\int_{0}^{T} g_{s}^{\varepsilon} \delta Z(s)-\int_{0}^{T} g_{s} \delta Z(s)\right|^{2} \leq 3\left(\mathbf{E}\left|\int_{0}^{T} g_{s}^{n, \varepsilon} \delta Z(s)-\int_{0}^{T} g_{s}^{n} \delta Z(s)\right|^{2}+\delta\right)
$$

and we can conclude by taking $\varepsilon \rightarrow 0$.

Acknowledgements. We would like to thank the anonymous referees for the careful reading of the manuscript and for valuable suggestions.

\section{REFERENCES}

[1] P. Abry and V. Pipiras, Wavelet-based synthesis of the Rosenblatt process. Signal Process. 86 (2006) 2326-2339.

[2] J.M.P. Albin, A note on the Rosenblatt distributions. Statist. Probab. Lett. 40 (1998) 83-91.

[3] J.M.P. Albin, On extremal theory for self similar processes. Ann. Probab. 26 (1998) 743-793.

[4] E. Alòs, O. Mazet and D. Nualart, Stochastic calculus with respect to Gaussian processes. Ann. Probab. 29 (2001) 766-801.

[5] E. Alòs and D. Nualart, Stochastic integration with respect to the fractional Brownian motion. Stoch. Stoch. Rep. 75 (2003) $129-152$.

[6] T. Androshuk and Y. Mishura, Mixed Brownian-fractional Brownian model: absence of arbitrage and related topics. Stochastics An Int. J. Probability Stochastic Processes 78 (2006) 281-300.

[7] F. Biagini, M. Campanino and S. Fuschini, Discrete approximation of stochastic integrals with respect of fractional Brownian motion of Hurst index $H>1 / 2$. Preprint University of Bologna (2005).

[8] P. Cheridito, H. Kawaguchi and M. Maejima, Fractional Ornstein-Uhlenbeck processes. Electron. J. Probab. 8 (2003) 1-14.

[9] L. Decreusefond and A.S. Ustunel, Stochastic analysis of the fractional Brownian motion. Potential Anal. 10 (1998) $177-214$.

[10] G. da Prato and J. Zabczyk, Stochastic equations in infinite dimensions. Cambridge University Press (1992).

[11] R.L. Dobrushin and P. Major, Non-central limit theorems for non-linear functionals of Gaussian fields. Z. Wahrscheinlichkeitstheorie verw. Gebiete 50 (1979) 27-52.

[12] A. Drewitz, Mild solutions to stochastic evolution equations with fractional Brownian motion. Diploma thesis at TU Darmstadt (2005).

[13] P. Embrechts and M. Maejima, Selfsimilar processes. Princeton University Press, Princeton, New York (2002).

[14] R. Fox and M.S. Taqqu, Multiple stochastic integrals with dependent integrators. J. Mult. Anal. 21 (1987) $105-127$.

[15] V. Goodman and J. Kuelbs, Gaussian chaos and functional law of the ierated logarithm for Itô-Wiener integrals. Ann. I.H.P., Section B 29 (1993) 485-512.

[16] M. Gradinaru, I. Nourdin, F. Russo and P. Vallois, $m$-order integrals and generalized Itôs formula; the case of a fractional Brownian motion with any Hurst parameter. Preprint, to appear in Annales de l'Institut Henri Poincaré (2003).

[17] M. Gradinaru, I. Nourdin and S. Tindel, Ito's and Tanaka's type formulae for the stochastic heat equation. J. Funct. Anal. 228 (2005) 114-143.

[18] P. Hall, W. Hardle, T. Kleinow and P. Schmidt, Semiparametric Bootstrap Approach to Hypothesis tests and Confidence intervals for the Hurst coefficient. Stat. Infer. Stoch. Process. 3 (2000) 263-276.

[19] M. Jolis and M. Sanz, Integrator properties of the Skorohod integral. Stochastics and Stochastics Reports 41 (1992) $163-176$.

[20] O. Kallenberg, On an independence criterion for multiple Wiener integrals. Ann. Probab. 19 (1991) 483-485.

[21] H. Kettani and J. Gubner, Estimation of the long-range dependence parameter of fractional Brownian motionin, in Proc. 28th IEEE LCNO3 (2003).

[22] I. Kruk, F. Russo and C.A. Tudor, Wiener integrals, Malliavin calculus and covariance measure structure. J. Funct. Anal. 249 (2007) 92-142. 
[23] N.N. Leonenko and V.V. Ahn, Rate of convergence to the Rosenblatt distribution for additive functionals of stochastic processes with long-range dependence. J. Appl. Math. Stoch. Anal. 14 (2001) 27-46.

[24] N.N. Leonenko and W. Woyczynski, Scaling limits of solutions of the heat equation for singular Non-Gaussian data. J. Stat. Phys. 91 423-438.

[25] M. Maejima and C.A. Tudor, Wiener integrals with respect to the Hermite process and a non central limit theorem. Stoch. Anal. Appl. 25 (2007) 1043-1056.

[26] O. Mocioalca and F. Viens, Skorohod integration and stochastic calculus beyond the fractional Brownian scale. J. Funct. Anal. $222(2004)$ 385-434.

[27] I. Norros, E. Valkeila and J. Virtamo, An elementary approach to a Girsanov formula and other analytical results for fractional Brownian motion. Bernoulli 5 (1999) 571-587.

[28] I. Nourdin, A simple theory for the study of SDEs driven by a fractional Brownian motion, in dimension one. Séminaire de Probabilités XLI (2006). To appear.

[29] D. Nualart, Malliavin Calculus and Related Topics. Springer (1995).

[30] D. Nualart and M. Zakai, Generalized mulptiple stochastic integrals and the representation of Wiener functionals. Stochastics 23 (1987) 311-330.

[31] V. Pipiras, Wavelet type expansion of the Rosenblatt process. J. Fourier Anal. Appl. 10 (2004) 599-634.

[32] V. Pipiras and M. Taqqu, Convergence of weighted sums of random variables with long range dependence. Stoch. Process. Appl. 90 (2000) 157-174.

[33] V. Pipiras and Murad Taqqu, Integration questions related to the fractional Brownian motion. Probab. Theor. Relat. Fields 118 (2001) 251-281.

[34] N. Privault and C.A. Tudor, Skorohod and pathwise stochastic calculus with respect to an $L^{2}$-process. Rand. Oper. Stoch. Equ. 8 (2000) 201-204.

[35] Z. Qian and T. Lyons, System control and rough paths. Clarendon Press, Oxford (2002).

[36] M. Rosenblatt, Independence and dependence. Proc. 4th Berkeley Symposium on Math, Stat. II (1961) 431-443.

[37] F. Russo and P. Vallois, Forward backward and symmetric stochastic integration. Probab. Theor. Relat. Fields 97 (1993) 403-421.

[38] F. Russo and P. Vallois, Stochastic calculus with respect to a finite quadratic variation process. Stoch. Stoch. Rep. 70 (2000) $1-40$.

[39] F. Russo and P. Vallois, Elements of stochastic calculus via regularization. Preprint, to appear in Séminaire de Probabilités (2006).

[40] G. Samorodnitsky and M. Taqqu, Stable Non-Gaussian random variables. Chapman and Hall, London (1994).

[41] A.S. Üstunel and M. Zakai, On independence and conditioning on Wiener space. Ann. Probab. 17 (1989) 1441-1453.

[42] M. Taqqu, Weak convergence to the fractional Brownian motion and to the Rosenblatt process. Z. Wahrscheinlichkeitstheorie verw. Gebiete 31 (1975) 287-302.

[43] M. Taqqu, Convergence of integrated processes of arbitrary Hermite rank. Z. Wahrscheinlichkeitstheorie verw. Gebiete $\mathbf{5 0}$ (1979) 53-83.

[44] M. Taqqu, A bibliographical guide to selfsimilar processes and long-range dependence. Dependence in Probability and Statistics, Birkhauser, Boston (1986) 137-162.

[45] S. Tindel, C.A. Tudor and F. Viens, Stochastic evolution equations with fractional Brownian motion. Probab. Theor. Relat. Fields. 127 (2003) 186-204.

[46] C.A. Tudor, Itô's formula for the infinite-dimensional fractional Brownian motion. J. Math. Kyoto University 45 (2005) $531-546$.

[47] W.B. Wu, Unit root testing for functionals of linear processes. Econ. Theory 22 (2005) 1-14. 